\title{
On a fractional $q$-differential inclusion on a time scale via endpoints and numerical calculations
}

\author{
Mohammad Esmael Samei ${ }^{1}$ and Shahram Rezapour ${ }^{2,3,4^{*}}$ (D)
}

\section{"Correspondence:}

shahramrezapour@duytan.edu.vn; sh.rezapour@mail.cmuh.org.tw; rezapourshahram@yahoo.ca; sh.rezapour@azaruniv.ac.ir ${ }^{2}$ Institute of Research and Development, Duy Tan University, Da Nang, 550000, Vietnam

${ }^{3}$ Faculty of Natural Sciences, Duy Tan University, Da Nang, 550000, Vietnam

Full list of author information is available at the end of the article

\begin{abstract}
By using an endpoint result for set-valued maps, we study the existence of solutions for a fractional $q$-differential inclusion with sum and integral boundary value conditions on the time scale $\mathbb{T}_{t_{0}}=\left\{t_{0} q, t_{0} q^{2}, \ldots\right\} \cup\{0\}$, where $t_{0}$ is a real number and $q \in(0,1)$. We provide an example involving some graphs and algorithms via numerical calculations to illustrate our main result.
\end{abstract}

MSC: Primary 34A08; 34B16; secondary 39A13

Keywords: Endpoint; q-differential inclusions; Sum boundary conditions; Caputo q-derivative; Integral boundary conditions

\section{Introduction}

In 1910, Jackson started the subject of $q$-difference equations [1]. The fractional calculus provides a meaningful generalization for the classical integration and differentiation to any order. Also, quantum calculus is equivalent to traditional infinitesimal calculus without the notion of limits. Despite the long history of these two theories, the investigation of their properties has remained untouched until recent time. In last decades, some researchers investigated $q$-fractional difference equations [2-5]. Later, $q$-fractional boundary value problems considered by many researchers (see for examples, [6-11]).

Plasma is also known as the ionized state of the matter. In this state of matter, plasma contains components such as free electrons, ions neutrals, and dust. The multicomponent plasmas deal with the partially or fully ionized state of plasma. It also fulfils the condition of quasi neutrality. The multi-component plasmas play a crucial role in plasma discharge and other industrial processing. The multi-component plasma has more than two components while the general plasma has ions and electrons. The wide range of plasmas itself give an opportunity to analyze such plasmas on distinct scales. There are two main areas of multi-component plasma: dusty plasma and the negative ions plasma. Both areas of multi-component plasma have a wide range of emerging applications in science and engineering. The time fractional Kersten-Krasil'shchik coupled KdV-mKdV nonlinear system and homogeneous two component time fractional coupled third order KdV systems are very important fractional nonlinear systems for describing the behavior of

(c) The Author(s) 2020. This article is licensed under a Creative Commons Attribution 4.0 International License, which permits use sharing, adaptation, distribution and reproduction in any medium or format, as long as you give appropriate credit to the original author(s) and the source, provide a link to the Creative Commons licence, and indicate if changes were made. The images or other third party material in this article are included in the article's Creative Commons licence, unless indicated otherwise in a credit line to the material. If material is not included in the article's Creative Commons licence and your intended use is not permitted by statutory regulation or exceeds the permitted use, you will need to obtain permission directly from the copyright holder. To view a copy of this licence, visit http://creativecommons.org/licenses/by/4.0/. 
waves in multi-component plasma and elaborate various nonlinear phenomena in plasma physics. In the past decades many researchers are used to various techniques for solving fractional nonlinear partial differential equation and find approximate and exact solutions of the fractional evolution equations (for more details, see [12-26]) and different applications of fractional calculus (see for example [27-30]).

In 2013, Ahmad et al. studied the fractional inclusion problem ${ }^{c} \mathcal{D}^{\beta}[u](t) \in T(t, u(t))$ with the integral boundary conditions $u^{j}(0)-c_{i} u^{j}(\delta)=a_{i} \int_{0}^{1} f_{j}(r, u(r)) \mathrm{d} r$ for $j=0,1,2$, where $T$ is a multifunction [31]. In 2014, Ghorbanian et al. reviewed the fractional differential inclusion problems

$$
\begin{aligned}
& { }^{c} \mathcal{D}^{\sigma_{1}}[z](t) \in T_{1}\left(t, z(t), z^{\prime}(t), z^{\prime \prime}(t)\right), \\
& { }^{c} \mathcal{D}^{\sigma_{2}}[z](t) \in T_{2}\left(t, z(t),{ }^{c} \mathcal{D}^{\beta_{1}}[z](t), \ldots,{ }^{c} \mathcal{D}^{\beta_{n}}[z](t)\right),
\end{aligned}
$$

with some integral boundary value conditions

$$
\left\{\begin{array}{l}
z(0)+z(\eta)+z(1)=\int_{0}^{1} f_{0}(r, z(r)) \mathrm{d} r, \\
{ }^{c} \mathcal{D}^{\zeta}[z](0)+{ }^{c} \mathcal{D}^{\zeta} z(\eta)+{ }^{c} \mathcal{D}^{\zeta}[z](1)=\int_{0}^{1} f_{1}(r, z(r)) \mathrm{d} r, \\
{ }^{c} \mathcal{D}^{\beta}[z](0)+{ }^{c} \mathcal{D}^{\beta}[z](\eta)+{ }^{c} \mathcal{D}^{\beta}[z](1)=\int_{0}^{1} f_{2}(r, z(r)) \mathrm{d} r,
\end{array}\right.
$$

and $z(0)+a z(1)=\sum_{i=1}^{n} \mathcal{I}^{\beta_{i}} z(\eta), Z^{\prime}(0)+b z^{\prime}(1)=\sum_{i=1}^{n}{ }^{c} \mathcal{D}^{\beta_{i}}[z](\eta)$, respectively, where $t \in J$, $2<\sigma_{1} \leq 3,1<\sigma_{2} \leq 2,0<\eta, \zeta, \beta_{i}<1,1<\beta<2, \sigma_{2}-\beta_{i} \geq 1$ for $1 \leq i \leq n, a>\sum_{i=1}^{n} \frac{\eta^{\beta_{i}+1}}{\Gamma\left(\beta_{i}+2\right)}$, $b>\sum_{i=1}^{n} \frac{\eta^{1-\beta_{i}}}{\Gamma\left(2-\beta_{i}\right)}, n \in \mathbb{N}, T_{1}: J \times \mathbb{R}^{3} \rightarrow P_{c p}(\mathbb{R}), T_{2}: J \times \mathbb{R}^{n+1} \rightarrow P_{c p}(\mathbb{R})$ are multifunctions, $f_{i}: J \times \mathbb{R} \rightarrow \mathbb{R}$ are continuous functions for $i=0,1,2$ and $P_{c p}(\mathbb{R})$ is the set of all compact subsets of $\mathbb{R}[32]$.

In 2015, Agarwal et al. investigated the fractional derivative inclusions ${ }^{c} \mathcal{D}^{\beta}[x](t) \in$ $F_{1}(t, x(t))$ and ${ }^{c} \mathcal{D}^{\beta}[x](t) \in F_{2}\left(t, x(t),{ }^{c} \mathcal{D}^{\zeta}[x](t)\right)$ with the boundary value problems $x(0)=$ $a \int_{0}^{v} x(\xi) \mathrm{d} \xi, x(1)=b \int_{0}^{\eta} x(\xi) \mathrm{d} \xi$ and $x(1)+x^{\prime}(1)=\int_{0}^{\eta} x(\xi) \mathrm{d} \xi, x(0)=0$, respectively, where $t \in J, \zeta, \eta, v \in(0,1), \beta \in(1,2]$ with $\beta-\zeta>1, a, b \in \mathbb{R},{ }^{c} \mathcal{D}^{\beta}$ is the Caputo differentiation and $F_{1}: J \times \mathbb{R} \times \mathbb{R} \rightarrow 2^{\mathbb{R}}, F_{2}: J \times \mathbb{R} \times \mathbb{R} \rightarrow 2^{\mathbb{R}}$ are compact valued multifunction [33]. Also, Ntouyas et al. studied the fractional inclusion problem ${ }^{c} \mathcal{D}_{0}^{\alpha}[u](t) \in$ $F\left(t, u(t), u^{\prime}(t),{ }^{c} \mathcal{D}_{0}^{p_{1}}[u](t), \ldots,{ }^{c} \mathcal{D}_{0}^{p_{k}}[u](t)\right)$ with sum and integral boundary conditions $u(0)+$ $\sum_{j=1}^{m} b_{j} u^{\prime \prime}(0)=0, \gamma_{1} u(\eta)+\gamma_{2} \int_{0}^{1} u(\tau) \mathrm{d} \tau=0$ and $\sum_{j=1}^{m} b_{j} u^{\prime}(1)+\gamma_{3} \int_{0}^{1} u(\tau) \mathrm{d} \tau=0$, where ${ }^{c} \mathcal{D}_{0}^{\alpha}$ denotes the Caputo fractional derivative of order $\alpha, t \in[0,1], 2<\alpha \leq 3,1<p_{i} \leq 2$, $(i=1, \ldots, k ; k \geq 1), 0<\eta<1, b_{j}(j=1, \ldots, m ; m \geq 1), \gamma_{1}, \gamma_{2}, \gamma_{3} \in \mathbb{R}$ and $F: J \times \mathbb{R}^{k+2} \rightarrow \mathcal{P}(\mathbb{R})$ is a compact-valued multifunction (citeNtouyasEtemad. In 2019, Samei et al. studied the hybrid Caputo-Hadamard fractional inclusion problem

$$
\left\{\begin{array}{l}
{ }_{H}^{C} \mathcal{D}^{\alpha}\left[\frac{x(t)-f\left(t, x(t), \mathcal{I}^{\beta_{1}}\left[h_{1}\right](t, x(t)), \mathcal{I}^{\beta_{2}}\left[h_{2}\right](t, x(t)), \ldots, \mathcal{I}^{\beta_{n}}\left[h_{n}\right](t, x(t))\right)}{g\left(t, x(t), \mathcal{I}^{\gamma}[x](t), \mathcal{I}^{\gamma}[x](t), \ldots, \mathcal{I}^{\prime} m[x](t)\right)}\right] \\
\quad \in K(t, x(t)), \\
x(1)=\mu(x), \quad x(e)=\eta(x),
\end{array}\right.
$$

where ${ }_{H}^{C} \mathcal{D}^{\alpha}$ and ${ }^{H} \mathcal{I}^{\alpha}$ denote the Caputo-Hadamard fractional derivative and Hadamard integral of order $\alpha$, respectively, $t \in J=[1, e], n, m \in \mathbb{N}, 1<\alpha \leq 2, \beta_{i}>0$ for $i=1,2, \ldots, n$, $\gamma_{i}>0$ for $i=1,2, \ldots, m$, the functions $f: J \times \mathbb{R}^{n+1} \rightarrow \mathbb{R}, g: J \times \mathbb{R}^{m+1} \rightarrow \mathbb{R} \backslash\{0\}, h_{i}: J \times$ 
$\mathbb{R} \rightarrow \mathbb{R}$ for $i=1,2, \ldots, n$, functions $\mu, \eta$ map $C(J, \mathbb{R})$ into $\mathbb{R}$ and the multifunction $K: J \times$ $\mathbb{R} \rightarrow P(\mathbb{R})$ satisfies certain conditions [35]. Also, Ntouyas et al. reviewed the multi-term nonlinear fractional $q$-integro-differential equation

$$
{ }^{c} \mathcal{D}_{q}^{\alpha}[x](t)=w\left(t, x(t),\left(\varphi_{1} x\right)(t),\left(\varphi_{2} x\right)(t),{ }^{c} \mathcal{D}_{q}^{\beta_{1}}[x](t),{ }^{c} \mathcal{D}_{q}^{\beta_{2}}[x](t), \ldots,{ }^{c} \mathcal{D}_{q}^{\beta_{n}}[x](t)\right)
$$

under some boundary conditions [36]. In 2019, Samei et al. discussed the fractional hybrid $q$-differential inclusions

$$
{ }^{c} \mathcal{D}_{q}^{\alpha}\left(\frac{k}{f\left(t, k, \mathcal{I}_{q}^{\alpha_{1}}[k], \ldots, \mathcal{I}_{q}^{\alpha_{n}}[k]\right)}\right) \in F\left(t, k, \mathcal{I}_{q}^{\beta_{1}}[k], \ldots, \mathcal{I}_{q}^{\beta_{k}}[k]\right),
$$

with the boundary conditions $k(0)=k_{0}$ and $k(1)=k_{1}$, where $1<\alpha \leq 2, q \in(0,1), k_{0}, k_{1} \in \mathbb{R}$, $\alpha_{i}>0$, for $i=1,2, \ldots, n, \beta_{j}>0$, for $j=1,2, \ldots, m, n, m \in \mathbb{N},{ }^{c} \mathcal{D}_{q}^{\alpha}$ denotes Caputo type $q$ derivative of order $\alpha, \mathcal{I}_{q}^{\beta}$ denotes the Riemann-Liouville type $q$-integral of order $\beta, f$ : $J \times \mathbb{R}^{n} \rightarrow(0, \infty)$ is continuous and $F: J \times \mathbb{R}^{m} \rightarrow P(\mathbb{R})$ is a multifunction [10].

By using the main idea of this work, we investigate the fractional $q$-differential inclusion

$$
{ }^{c} \mathcal{D}_{q}^{\sigma}[k](t) \in \mathcal{T}\left(t, k(t), k^{\prime}(t),{ }^{c} \mathcal{D}_{q}^{\zeta 1}[k](t), \ldots,{ }^{c} \mathcal{D}_{q}^{\zeta m}[k](t)\right),
$$

with sum and integral boundary value conditions

$$
\left\{\begin{array}{l}
k(0)+\Sigma k^{\prime \prime}(0)=0, \\
a_{1} k(\tau)+a_{2} \varrho(1)=0, \\
\Sigma k^{\prime}(1)+a_{3} \varrho(1)=0,
\end{array}\right.
$$

where $2<\sigma \leq 3, t \in \bar{J}:=[0,1],{ }^{c} \mathcal{D}_{q}^{\sigma}$ denotes the Caputo fractional $q$-derivative of order $\sigma, 1<\zeta_{i} \leq 2(i=1, \ldots, m), 0<\tau<1, \Sigma=\sum_{j=1}^{k} c_{j}$ with $c_{j} \in \mathbb{R},(j=1, \ldots, m), \varrho, \phi:[0, \infty) \rightarrow$ $[0, \infty)$ define by $\varrho(v)=\int_{0}^{v} \phi(k(r)) \mathrm{d} r, a_{1}, a_{2}, a_{3} \in \mathbb{R}$ and $\mathcal{T}: \bar{J} \times \mathbb{R}^{m+2} \rightarrow \mathcal{P}(\mathbb{R})$ is a compactvalued multifunction.

This work is arranged as: In Sect. 2, we state some useful definitions and lemma on the fundamental concepts of $q$-fractional calculus and multifunctions. In Sect. 3, some main theorems on the solutions of fractional $q$-differential inclusion (1)-(2) are stated. Section 4 contains an illustrative example to show the validity and applicability of our results. The paper concludes with some interesting observations. In Sect. 5, conclusions are presented.

\section{Essential preliminaries}

Throughout this article, we shall apply the time scales calculus notation [37]. In fact, we consider the fractional $q$-calculus on the time scale $\mathbb{T}_{t_{0}}=\{0\} \cup\left\{t: t=t_{0} q^{n}\right\}$, where $n \geq 0$, $t_{0} \in \mathbb{R}$ and $q \in(0,1)$. Let $a \in \mathbb{R}$. Define $[a]_{q}=\frac{1-q^{a}}{1-q}[1]$. The power function $(x-y)_{q}^{n}$ with $n \in \mathbb{N}_{0}$ is defined by $(x-y)_{q}^{(n)}=\prod_{k=0}^{n-1}\left(x-y q^{k}\right)$ for $n \geq 1$ and $(x-y)_{q}^{(0)}=1$, where $x$ and $y$ are real numbers and $\mathbb{N}_{0}:=\{0\} \cup \mathbb{N}[2]$. Also, for $\alpha \in \mathbb{R}$ and $a \neq 0$, we have

$$
(x-y)_{q}^{(\alpha)}=x^{\alpha} \prod_{k=0}^{\infty} \frac{x-y q^{k}}{x-y q^{\alpha+k}}
$$



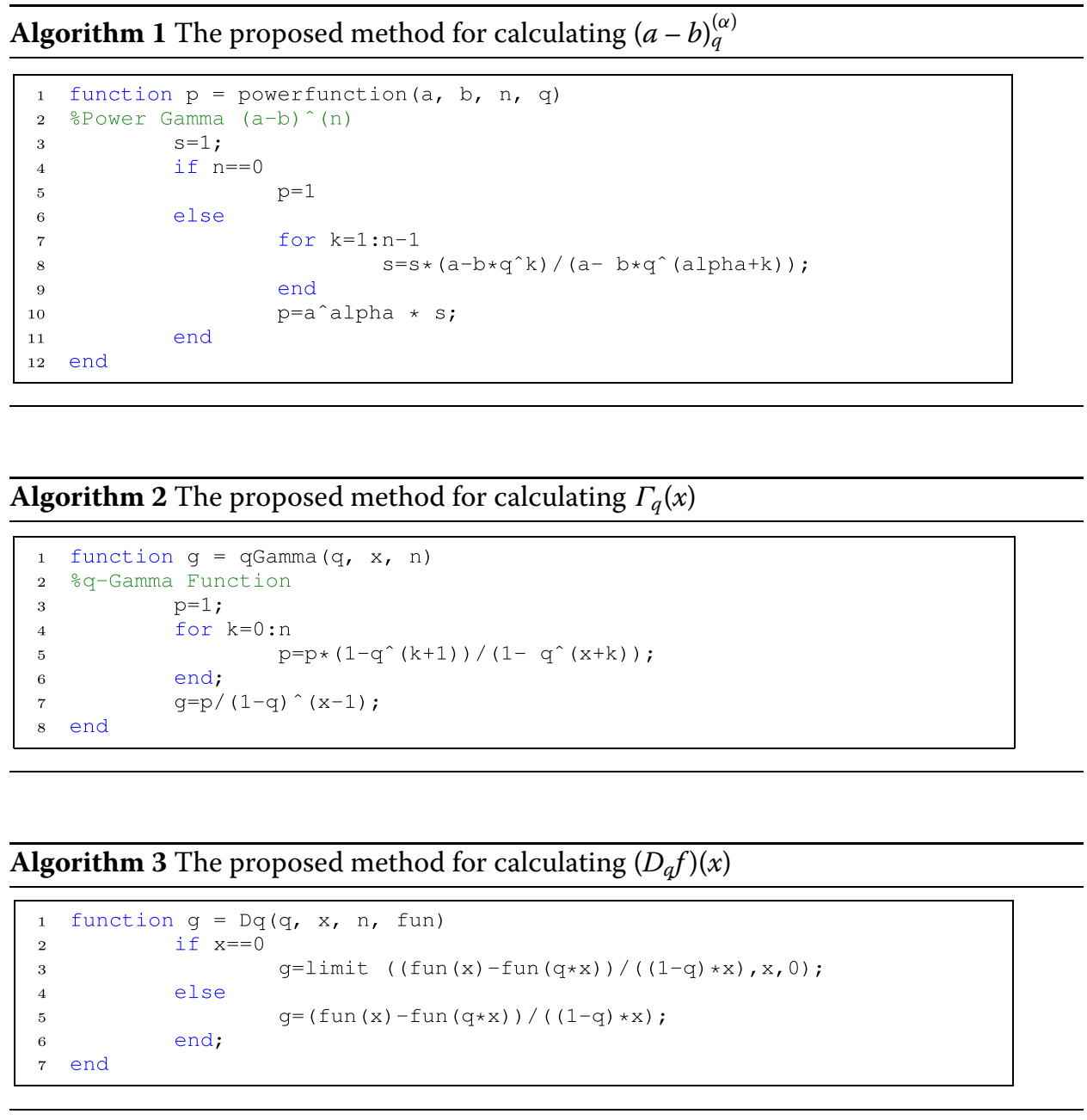

If $y=0$, then it is clear that $x^{(\alpha)}=x^{\alpha}$ [7] (see the Algorithm 1). The $q$-Gamma function is given by $\Gamma_{q}(z)=(1-q)^{(z-1)} /(1-q)^{z-1}$, where $z \in \mathbb{R} \backslash\{0,-1,-2, \ldots\}$ [1]. Note that, $\Gamma_{q}(z+1)=[z]_{q} \Gamma_{q}(z)$. The Algorithm 2 shows a pseudo-code description of the technique for estimating $q$-Gamma function of order $n$. The $q$-derivative of function $f$, is defined by $\left(\mathcal{D}_{q} f\right)(x)=\frac{f(x)-f(q x)}{(1-q) x}$ and $\left(\mathcal{D}_{q} f\right)(0)=\lim _{x \rightarrow 0}\left(\mathcal{D}_{q} f\right)(x)$ which is shown in Algorithm 3 [2,3]. Furthermore, the higher order $q$-derivative of a function $f$ is defined by $\left(D_{q}^{n} f\right)(x)=D_{q}\left(D_{q}^{n-1} f\right)(x)$ for $n \geq 1$, where $\left(D_{q}^{0} f\right)(x)=f(x)[2,3]$. The $q$-integral of a function $f$ is defined on $[0, b]$ by $I_{q} f(x)=\int_{0}^{x} f(s) \mathrm{d}_{q} s=x(1-q) \sum_{k=0}^{\infty} q^{k} f\left(x q^{k}\right)$ for $0 \leq x \leq b$, provided the series is absolutely converges [2, 3]. If $x$ in [0,T], then

$$
\int_{x}^{T} f(r) \mathrm{d}_{q} r=I_{q} f(T)-I_{q} f(x)=(1-q) \sum_{k=0}^{\infty} q^{k}\left[T f\left(T q^{k}\right)-x f\left(x q^{k}\right)\right]
$$

whenever the series exists. In addition, we can interchange the order of double $q$-integral by $\int_{0}^{t} \int_{0}^{s} h(r) \mathrm{d}_{q} r \mathrm{~d}_{q} s=\int_{0}^{t} \int_{q r}^{t} h(r) \mathrm{d}_{q} s \mathrm{~d}_{q} r$ [38]. Actually the interchange of order is true, since

$$
\int_{0}^{t} \int_{q r}^{t} h(s) \mathrm{d}_{q} s \mathrm{~d}_{q} r=\int_{0}^{t}(t-q r)^{(\sigma-1)} h(r) \mathrm{d}_{q} r
$$




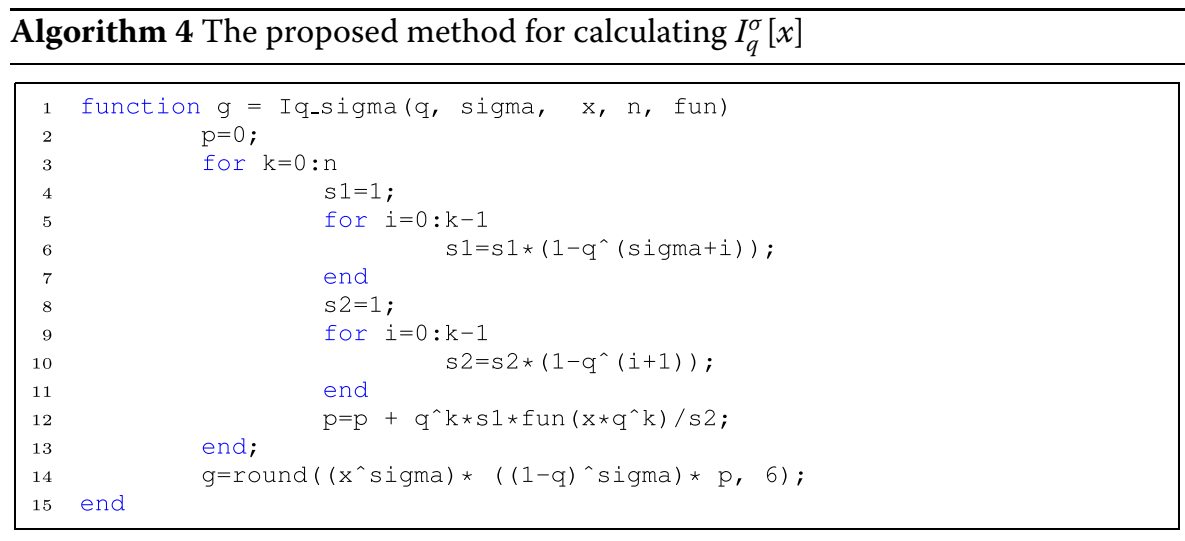

$$
\begin{aligned}
& =t(1-q) \sum_{i=0}^{\infty} q^{i} h\left(q^{i} t\right)\left(t-q^{i+1} t\right) \\
& =t^{2}(1-q)^{2} \sum_{i=0}^{\infty} q^{i} h\left(q^{i} t\right)\left(\sum_{i=0}^{\infty} q^{i}\right) .
\end{aligned}
$$

In addition the left side can be written as

$$
\begin{aligned}
\int_{0}^{t} \int_{0}^{r} h(s) \mathrm{d}_{q} s \mathrm{~d}_{q} r & =t(1-q) \sum_{i=0}^{\infty} q^{i} \int_{0}^{t q^{i}} h(r) \mathrm{d}_{q} r \\
& =t^{2}(1-q)^{2} \sum_{i=0}^{\infty} \sum_{j=0}^{\infty} q^{i+2 j} h\left(q^{i+j} t\right) .
\end{aligned}
$$

The operator $I_{q}^{n}$ is given by $\left(I_{q}^{0} h\right)(x)=h(x)$ and $\left(I_{q}^{n} h\right)(x)=\left(I_{q}\left(I_{q}^{n-1} h\right)\right)(x)$ for all $n \geq 1$ and $h \in C([0, T])[2,3]$. It has been proved that $\left(D_{q}\left(I_{q} h\right)\right)(x)=h(x)$ and $\left(I_{q}\left(D_{q} h\right)\right)(x)=h(x)-h(0)$ whenever $h$ is continuous at $x=0[2,3]$. The fractional Riemann-Liouville type $q$-integral of the function $h$ on $J=(0,1)$ for $\sigma \geq 0$ is defined by $\mathcal{I}_{q}^{0}[h](t)=h(t)$ and

$$
\begin{aligned}
\mathcal{I}_{q}^{\sigma}[h](t) & =\frac{1}{\Gamma_{q}(\sigma)} \int_{0}^{t}(t-q r)^{(\sigma-1)} h(r) \mathrm{d}_{q} r \\
& =t^{\sigma}(1-q)^{\sigma} \sum_{k=0}^{\infty} q^{k} \frac{\prod_{i=1}^{k-1}\left(1-q^{\sigma+i}\right)}{\prod_{i=1}^{k-1}\left(1-q^{i+1}\right)} h\left(t q^{k}\right)
\end{aligned}
$$

for $t \in J$ [39]. Also, the Caputo fractional $q$-derivative of a function $h$ is defined by

$$
{ }^{c} \mathcal{D}_{q}^{\sigma}[h](t)=\mathcal{I}_{q}^{[\sigma]-\sigma}\left[\mathcal{D}_{q}^{[\sigma]}[h]\right](t)=\frac{1}{\Gamma_{q}([\sigma]-\sigma)} \int_{0}^{t}(t-q r)^{([\sigma]-\sigma-1)} \mathcal{D}_{q}^{[\sigma]}[h](r) \mathrm{d}_{q} r
$$

where $t \in J$ and $\sigma>0$ [39]. It has been proved that $\mathcal{I}_{q}^{\beta}\left[\mathcal{I}_{q}^{\alpha}[h]\right](x)=\mathcal{I}_{q}^{\alpha+\beta}[h](x)$ and $\mathcal{D}_{q}^{\alpha}\left[\mathcal{I}_{q}^{\alpha}[h]\right](x)=h(x)$, where $\alpha, \beta \geq 0$ [39]. The Algorithm 4 shows pesudo-code $\mathcal{I}_{q}^{\alpha}[h](x)$.

Let $(\mathcal{E}, \rho)$ be a metric space. Denote by $\mathcal{P}(\mathcal{E})$ and $2^{\mathcal{E}}$ the class of all subsets and the class of all nonempty subsets of $\mathcal{E}$, respectively. Thus, $\mathcal{P}_{c l}(\mathcal{E}), \mathcal{P}_{b d}(\mathcal{E}), \mathcal{P}_{c \nu}(\mathcal{E})$ and $\mathcal{P}_{c p}(\mathcal{E})$ denote the classes of all closed, bounded, convex and compact subsets of $\mathcal{E}$, respectively. A mapping $\mathcal{T}: \mathcal{E} \rightarrow 2^{\mathcal{E}}$ is called a multifunction on $\mathcal{E}$ and $e \in \mathcal{E}$ is called a fixed point of $\mathcal{T}$ 
whenever $e \in \mathcal{T}(e)$. An element $e \in \mathcal{E}$ is called an endpoint of a multifunction $\mathcal{T}: \mathcal{E} \rightarrow 2^{\mathcal{E}}$ whenever $\mathcal{T}(e)=\{e\}[40]$. Also, we say that $\mathcal{T}$ has an approximate endpoint property whenever $\inf _{e \in \mathcal{E}} \sup _{f \in \mathcal{T}(e)} \rho(e, f)=0$ [40]. A function $\psi: \mathbb{R} \rightarrow \mathbb{R}$ is called upper semi-continuous whenever $\lim \sup _{n \rightarrow \infty} \psi\left(r_{n}\right) \leq \psi(r)$ for all sequence $\left\{r_{n}\right\}_{n \geq 1}$ with $r_{n} \rightarrow r$.

A multifunction $\mathcal{T}: \mathcal{E} \rightarrow \mathcal{P}_{c l}(\mathcal{E})$ is said to be lower semi-continuous whenever for every open set $\mathcal{O}$ of $\mathcal{E}$, the set $\mathcal{T}^{-1}(\mathcal{O}):=\{z \in \mathcal{E}: \mathcal{T}(z) \cap \mathcal{O} \neq \emptyset\}$ is open [41]. We say that $\mathcal{T}$ is upper semi-continuous whenever the set $\{z \in \mathcal{X}: \mathcal{T}(z) \subset \mathcal{O}\}$ is open for any open set $\mathcal{O}$ of $\mathcal{E}$ [41]. Also, $\mathcal{T}: \mathcal{E} \rightarrow \mathcal{P}_{c p}(\mathcal{E})$ is called compact if $\overline{\mathcal{T}(\mathcal{B})}$ is a compact set of $\mathcal{E}$ for any bounded subsets $\mathcal{B}$ of $\mathcal{E}$ [41]. A multifunction $\mathcal{T}: \bar{J} \rightarrow \mathcal{P}_{c l}(\mathbb{R})$ is said to be measurable whenever the function $t \mapsto \rho(y, \mathcal{T}(t))=\inf \{|y-z|: z \in \mathcal{T}(t)\}$ is measurable for all $y \in \mathbb{R}$, $\bar{J}=[0,1][41]$. Define the Pompeiu-Hausdorff metric $P_{\rho}: 2^{\mathcal{E}} \times 2^{\mathcal{E}} \rightarrow[0, \infty)$ by

$$
P_{\rho}(S, T)=\max \left\{\sup _{s \in S} \rho(s, T), \sup _{t \in T} \rho(S, t)\right\}
$$

where $\rho(S, t)=\inf _{s \in S} \rho(s ; t)$. Then $\left(\mathcal{P}_{b, c l}(\mathcal{E}), P_{\rho}\right)$ is a metric space and $\left(\mathcal{P}_{c l}(\mathcal{E}), P_{\rho}\right)$ is a generalized metric space [41]. A multifunction $\mathcal{T}: \mathcal{E} \rightarrow \mathcal{P}_{c l}(\mathcal{E})$ is called a $\lambda$-contraction whenever there exists $\lambda \in J=(0,1)$ such that $P_{\rho}\left(\mathcal{T}\left(e_{1}\right), \Theta\left(e_{2}\right)\right) \leq \lambda \rho\left(e_{1}, e_{2}\right)$ for all $e_{1}, e_{2} \in$ $\mathcal{E}$ [42]. In 1970, Covitz and Nadler proved that each closed valued contractive multifunction on a complete metric space has a fixed point [42]. We say that $\mathcal{T}: \bar{J} \times \mathbb{R}^{2 m} \rightarrow$ $2^{\mathbb{R}}$ is a Carathéodory multifunction whenever $t \mapsto \mathcal{T}\left(t, r_{1}, \ldots, r_{2 m}\right)$ is measurable for all $r_{1}, \ldots, r_{2 m} \in \mathbb{R}$ and $\left(r_{1}, \ldots, r_{2 m}\right) \mapsto \mathcal{T}\left(t, r_{1}, \ldots, r_{2 m}\right)$ is an upper semi-continuous map for almost all $t \in \bar{J}[41,43,44]$. Also, a Carathéodory multifunction $\mathcal{T}: \bar{J} \times \mathbb{R}^{2 m} \rightarrow 2^{\mathbb{R}}$ is called $L^{1}$-Carathéodory whenever for each $\eta>0$ there exists $\Upsilon_{\eta} \in L^{1}\left(\bar{J}, \mathbb{R}^{+}\right)$such that

$$
\left\|\mathcal{T}\left(t, r_{1}, \ldots, r_{2 m}\right)\right\|=\sup \left\{|k|: k \in \mathcal{T}\left(t, r_{1}, \ldots, r_{2 m}\right)\right\} \leq \Upsilon_{\eta}(t)
$$

for all $\left|r_{1}\right|, \ldots,\left|r_{2 m}\right| \leq \eta$ and for almost all $t \in \bar{J}[41,43,44]$. For each $i$, define the space $E_{i}=\left\{k(t): k(t), k^{\prime}(t),{ }^{c} \mathcal{D}_{q}^{\zeta_{i}}[k](t) \in \mathcal{A}\right\}$ endowed with the norm

$$
\|k\|_{i}=\sup _{t \in \bar{J}}|k(t)|+\sup _{t \in \bar{J}}\left|k^{\prime}(t)\right|+\sup _{t \in \bar{J}}\left|{ }^{c} \mathcal{D}_{q}^{\zeta_{i}}[k](t)\right|
$$

where $\mathcal{A}=C(\bar{J}, \mathbb{R})$. Also, consider the product space $\mathcal{E}=E_{1} \times \cdots \times E_{m}$ endowed with the norm $\left\|\left(k_{1}, \ldots, k_{m}\right)\right\|=\sum_{i=1}^{m}\left\|k_{i}\right\|_{i}$. Then $(\mathcal{E},\|\cdot\|)$ is a Banach space [45]. By using the idea of $[31,34,46]$, define the set of the selections of $\mathcal{S}$, at $k$ by

$$
S_{\mathcal{T}, k}=\left\{p \in L^{1}(\bar{J}): p(t) \in \mathcal{T}\left(t, k(t), k^{\prime}(t),{ }^{c} \mathcal{D}_{q}^{\zeta_{1}}[k](t), \ldots,{ }^{c} \mathcal{D}_{q}^{\zeta_{m}}[k](t)\right)\right\}
$$

for all $t \in \bar{J}, k=\left(k_{1}, \ldots, k_{m}\right) \in \mathcal{E}$ and $1 \leq i \leq m$. One can check that $S_{\mathcal{T}, k} \neq \emptyset$ for all $k \in \mathcal{E}$ whenever $\operatorname{dim} \mathcal{E}<\infty$ [47]. For the proof of our main result we use the following endpoint fixed point theorem of Amini-Harandi [40].

Lemma 1 ([40]) Let $\psi:[0, \infty) \rightarrow[0, \infty)$ be an upper semi-continuous function such that $\psi(s)<s$ and $\liminf _{s \rightarrow \infty}(s-\psi(s))>0$ for all $s>0,(\mathcal{E}, \rho)$ a complete metric space and $\mathcal{T}$ : $\mathcal{E} \rightarrow \mathcal{T}_{\text {cl,bd }}(\mathcal{E})$ a multifunction such that $P_{\rho}(\mathcal{T}(e), \mathcal{T}(f)) \leq \psi(\rho(e, f))$ for all e,f $\in \mathcal{E}$. Then $\mathcal{T}$ has a unique endpoint if and only if $\mathcal{T}$ has approximate endpoint property. 


\section{Main results}

Now, we are ready to provide our main results.

Lemma 2 Let $z(t) \in \mathcal{A}$ and $\sigma \in(2,3]$. Then the unique solution of the fractional problem ${ }^{c} \mathcal{D}_{q}^{\sigma}[k](t)=z(t)$ under boundary value conditions $k(0)+\Sigma k^{\prime \prime}(0)=0, a_{1} k(\tau)+a_{2} \varrho(1)=0$ and $\Sigma k^{\prime}(1)+a_{3} \varrho(1)=0$ is given by

$$
\begin{aligned}
k(t)= & \mathcal{I}_{q}^{\sigma}[z](t)+\Delta a_{1} A_{1}(t) \mathcal{I}_{q}^{\sigma}[z](\tau) \\
& +\Delta\left(a_{2} A_{1}(t)+a_{3} A_{2}(t)\right) \mathcal{I}_{q}^{\sigma}\left[\mathcal{I}_{q}^{\sigma}[z](s)\right](1)+\Delta A_{2}(t) \Sigma \mathcal{I}_{q}^{\sigma-1}[z](1)
\end{aligned}
$$

where $\mathcal{I}_{q}^{\sigma}\left[\mathcal{I}_{q}^{\sigma}[z](s)\right](1)$ is defined by (3), $\Sigma=\sum_{j=1}^{k} c_{j}$ with $c_{1}, \ldots, c_{k} \in \mathbb{R}, \varrho:[0, \infty) \rightarrow[0, \infty)$ define by $\varrho(v)=\int_{0}^{v} k(r) \mathrm{d} r$,

$$
\begin{aligned}
\Delta= & {\left[\left(a_{1} \tau+\frac{a_{2}}{2}\right)\left(2 \Sigma+\frac{a_{3}}{3}-2 a_{3} \Sigma\right)\right.} \\
& \left.+\left(\Sigma++\frac{a_{3}}{2}\right)\left(2 \Sigma\left(a_{1}+a_{2}\right)-\left(a_{1} \tau^{2}+\frac{a_{2}}{3}\right)\right)\right]^{-1} \neq 0
\end{aligned}
$$

and

$$
\begin{aligned}
& A_{1}(t)=\left(\Sigma+\frac{a_{3}}{2}\right)\left(t^{2}-t-2 \Sigma\right)+2 a_{3} t \Sigma \\
& A_{2}(t)=\left(a_{1} \tau+\frac{a_{2}}{2}\right)\left(2 \Sigma-t^{2}\right)+t\left(a_{1} \tau^{2}+\frac{a_{2}}{3}-2\left(a_{1}+a_{2}\right) \Sigma\right)
\end{aligned}
$$

Proof It is known that the solution of the fractional $q$-differential equation ${ }^{c} \mathcal{D}^{\sigma} k(t)=z(t)$ is

$$
k(t)=\mathcal{I}_{q}^{\sigma}[z](t)+d_{0}+d_{1} t+d_{2} t^{2}
$$

where $d_{0}, d_{1}$ are real constants and $t \in \bar{J}$ [48]. Thus, we have $k^{\prime}(t)=\mathcal{I}_{q}^{\sigma-1}[z](t)+d_{1}+2 d_{2} t$ and $k^{\prime \prime}(t)=\mathcal{I}_{q}^{\sigma-2}[z](t)+2 d_{2}$. By using the boundary conditions, we obtain $d_{0}+2 d_{2} \Sigma=0$,

$$
\begin{gathered}
\left(a_{1}+a_{2}\right) d_{0}+\left(a_{1} \tau+\frac{a_{2}}{2}\right) d_{1}+\left(a_{1} \tau^{2}+\frac{a_{2}}{3}\right) d_{2} \\
=-a_{2} \mathcal{I}_{q}^{\sigma}\left[\mathcal{I}_{q}^{\sigma}[z](s)\right](1)-a_{1} \mathcal{I}_{q}^{\sigma}[z](\tau)
\end{gathered}
$$

and

$$
a_{3} d_{0}+\left(\Sigma+\frac{a_{3}}{2}\right) d_{1}+\left(2 \Sigma+\frac{a_{3}}{3}\right) d_{2}=-a_{3} \mathcal{I}_{q}^{\sigma}\left[\mathcal{I}_{q}^{\sigma}[z](s)\right](1)-\Sigma \mathcal{I}_{q}^{\sigma-1}[z](1)
$$

Hence by an easy calculation, we get

$$
\begin{aligned}
d_{0}= & 2 \Sigma \Delta\left[-\left(\Sigma+\frac{a_{3}}{2}\right)\left(a_{2} \mathcal{I}_{q}^{\sigma}\left[\mathcal{I}_{q}^{\sigma}[z](s)\right](1)+a_{1} \mathcal{I}_{q}^{\sigma}[z](\tau)\right)\right. \\
& \left.+\left(a_{1} \tau+\frac{a_{2}}{2}\right)\left(a_{3} \mathcal{I}_{q}^{\sigma}\left[\mathcal{I}_{q}^{\sigma}[z](s)\right](1)+\Sigma \mathcal{I}_{q}^{\sigma-1}[z](1)\right)\right]
\end{aligned}
$$




$$
\begin{aligned}
d_{1}= & \Delta\left[\left(2 a_{3} \Sigma-\Sigma-\frac{a_{3}}{3}\right)\left(a_{2} \mathcal{I}_{q}^{\sigma}\left[\mathcal{I}_{q}^{\sigma}[z](s)\right](1)+a_{1} \mathcal{I}_{q}^{\sigma}[z](\tau)\right)\right. \\
& \left.+\left(a_{1} \tau^{2}+\frac{a_{2}}{3}-2 \Sigma\left(a_{1}+a_{2}\right)\right)\left(a_{3} \mathcal{I}_{q}^{\sigma}\left[\mathcal{I}_{q}^{\sigma}[z](s)\right](1)+\Sigma \mathcal{I}_{q}^{\sigma-1}[z](1)\right)\right] \\
d_{2}= & \Delta\left[\left(\Sigma+\frac{a_{3}}{2}\right)\left(a_{2} \mathcal{I}_{q}^{\sigma}\left[\mathcal{I}_{q}^{\sigma}[z](s)\right](1)+a_{1} \mathcal{I}_{q}^{\sigma}[z](\tau)\right)\right. \\
& \left.-\left(a_{1} \tau+\frac{a_{2}}{2}\right)\left(a_{3} \mathcal{I}_{q}^{\sigma}\left[\mathcal{I}_{q}^{\sigma}[z](s)\right](1)+\Sigma \mathcal{I}_{q}^{\sigma-1}\right)\right] .
\end{aligned}
$$

Now, substituting the values of $d_{0}, d_{1}$ and $d_{2}$ in (7), we obtain (4).

From definition (6), we can see that

$$
\begin{aligned}
& \left|A_{1}(t)\right| \leq 2\left(|\Sigma|+\frac{\left|a_{3}\right|}{2}\right)(1+|\Sigma|)+2\left|a_{3}\right||\Sigma|:={ }_{0} A_{1}, \\
& \left|A_{1}^{\prime}(t)\right| \leq\left(2\left|a_{3}\right|+1\right)|\Sigma|+\frac{\left|a_{3}\right|}{2}:={ }_{0} A_{1}^{\prime}, \\
& \left|A_{2}(t)\right| \leq\left(\left|a_{1}\right| \tau+\frac{\left|a_{2}\right|}{2}\right)(2|\Sigma|+1)+\left|a_{1}\right| \tau^{2}+\frac{\left|a_{2}\right|}{3}+2\left|a_{1}+a_{2}\right||\Sigma|:={ }_{0} A_{2}, \\
& \left|A_{2}^{\prime}(t)\right| \leq 2\left(\left|a_{1}\right| \tau+\frac{\left|a_{2}\right|}{2}\right)+\left|a_{1}\right| \tau^{2}+\frac{\left|a_{2}\right|}{3}+2\left|a_{1}+a_{2}\right||\Sigma|:={ }_{0} A_{2}^{\prime} .
\end{aligned}
$$

Also,

$$
{ }^{c} \mathcal{D}_{q}^{\zeta_{i}}\left[A_{1}\right](t)=\left(\Sigma+\frac{a_{3}}{2}\right)\left[-\frac{1}{\Gamma_{q}\left(2-\zeta_{i}\right)} t^{1-\zeta_{i}}+\frac{2}{\Gamma_{q}\left(3-\zeta_{i}\right)} t^{2-\zeta_{i}}\right]+\frac{2 a_{3} \Sigma}{\Gamma_{q}\left(2-\zeta_{i}\right)} t^{1-\zeta_{i}}
$$

and

$$
\begin{aligned}
{ }^{c} \mathcal{D}_{q}^{\zeta_{i}}\left[A_{2}\right](t)= & \left(a_{1} \tau+\frac{a_{2}}{2}\right)\left(-\frac{2}{\Gamma_{q}\left(3-\zeta_{i}\right)} t^{2-\zeta_{i}}\right) \\
& +\left(a_{1} \tau^{2}+\frac{a_{2}}{3}-2\left(a_{1}+a_{2}\right) \Sigma\right) \frac{1}{\Gamma_{q}\left(2-\zeta_{i}\right)} t^{1-\zeta_{i}}
\end{aligned}
$$

for $t \in \bar{J}$ and $i=1, \ldots, m$. From this we obtain

$$
\begin{aligned}
\left|{ }^{c} \mathcal{D}_{q}^{\zeta_{i}}\left[A_{1}\right](t)\right| \leq & \left(\Sigma+\frac{\left|a_{3}\right|}{2}\right)\left[\frac{1}{\Gamma_{q}\left(2-\zeta_{i}\right)}+\frac{2}{\Gamma_{q}\left(3-\zeta_{i}\right)}\right] \\
& +2\left|a_{3}\right||\Sigma| \frac{1}{\Gamma_{q}\left(2-\zeta_{i}\right)}:={ }_{i} A_{1}, \\
\left|{ }^{c} \mathcal{D}_{q}^{\zeta_{i}}\left[A_{2}\right](t)\right| \leq & \left(\left|a_{1}\right| \tau+\frac{\left|a_{2}\right|}{2}\right) \frac{2}{\Gamma_{q}\left(3-\zeta_{i}\right)} \\
& +\left(\left|a_{1}\right| \tau^{2}+\frac{\left|a_{2}\right|}{3}+2\left|a_{1}+a_{2}\right||\Sigma|\right) \frac{1}{\Gamma_{q}\left(2-\zeta_{i}\right)}:={ }_{i} A_{2} .
\end{aligned}
$$

Definition 3 A function $\left(k_{1}, k_{2}, \ldots, k_{m}\right)$ belongs to $\prod_{i=1}^{m} A C^{1}(\bar{J})$ is a solution for the fractional $q$-differential inclusion (1) whenever it satisfies the boundary value conditions and 
there exists a function $\left(z_{1}, z_{2}, \ldots, z_{m}\right) \in \prod_{i=1}^{m} L^{1}(\bar{J})$ such that

$$
z_{i}(t) \in \mathcal{T}\left(t, k(t), k^{\prime}(t),{ }^{c} \mathcal{D}_{q}^{\zeta 1}[k](t), \ldots,{ }^{c} \mathcal{D}_{q}^{\zeta m}[k](t)\right)
$$

and

$$
\begin{aligned}
k(t)= & \mathcal{I}_{q}^{\sigma}[z](t)+\Delta a_{1} A_{1}(t) \mathcal{I}_{q}^{\sigma}[z](\tau) \\
& +\Delta\left(a_{2} A_{1}(t)+a_{3} A_{2}(t)\right) \mathcal{I}_{q}^{\sigma}\left[\mathcal{I}_{q}^{\sigma}[z](s)\right](1)+\Delta A_{2}(t) \Sigma \mathcal{I}_{q}^{\sigma-1}[z](1),
\end{aligned}
$$

for $t \in \bar{J}$ and $1 \leq i \leq m$, where $A_{1}(t)$ and $A_{2}(t)$ are defined in (6).

Theorem 4 Suppose that $\mathcal{T}: \bar{J} \times \mathbb{R}^{m+2} \rightarrow \mathcal{P}_{c p}(\mathbb{R})$ is an integrable bounded multifunction such that $t \vdash \mathcal{T}\left(t, x_{1}, x_{2}, v_{1}, \ldots, v_{m}\right)$ is measurable, $\psi:[0, \infty) \rightarrow[0, \infty)$ is a nondecreasing upper semi-continuous mapping such that $\liminf _{t \rightarrow \infty}(t-\psi(t))>0$ and $\psi(t)<t$ for all $t>0$ and there exist continuous functions $\gamma: \bar{J} \rightarrow[0, \infty)$ such that

$$
P_{\rho}\left(\mathcal{T}\left(t, k_{1}, \ldots, k_{1}, k_{2}, \ldots, k_{m+2}\right), \mathcal{T}\left(t, k_{1}^{\prime}, k_{2}^{\prime}, \ldots, u_{m+2}^{\prime}\right)\right) \leq \Xi \gamma(t) \sum_{i=1}^{m+2}\left(\left|k_{i}-k_{i}^{\prime}\right|\right),
$$

for all $t \in \bar{J},\left(k_{1} k_{2}, \ldots, k_{m+2}\right) \in \mathcal{E}, x_{1}, x_{2}$ and $v_{i} \in \mathbb{R}$ for $1 \leq i \leq m+2$, where

$$
\begin{aligned}
\Xi= & \frac{1}{M_{1}+M_{2}+\sum_{i=1}^{m}{ }_{i} M_{3}}, \\
M_{1}= & \|\gamma\|\left[\frac{1}{\Gamma_{q}(\sigma+1)}+\frac{\left|a_{1}\right|_{0} A_{1} \tau^{\sigma}|\Delta|}{\Gamma_{q}(\sigma+1)}\right. \\
& \left.+\frac{\left(\left|a_{2}\right|_{0} A_{1}+\left|a_{3}\right|_{0} A_{2}\right)|\Delta|}{\Gamma_{q}(\sigma+2)}+\frac{{ }_{0} A_{2}|\Delta|}{\Gamma_{q}(\sigma)}|\Sigma|\right], \\
M_{2}= & \|\gamma\|\left[\frac{1}{\Gamma_{q}(\sigma-1)}+\frac{\left|a_{1}\right|_{0} A_{1}^{\prime} \tau^{\sigma}|\Delta|}{\Gamma_{q}(\sigma+1)}\right. \\
& \left.+\frac{\left(\left|a_{2}\right|{ }_{0} A_{1}^{\prime}+\left|a_{3}\right|_{0} A_{2}^{\prime}\right)|\Delta|}{\Gamma_{q}(\sigma+2)}+\frac{{ }_{0} A_{2}^{\prime}|\Delta|}{\Gamma_{q}(\sigma)}|\Sigma|\right], \\
{ }_{i} M_{3}= & \|\gamma\|\left[\frac{1}{\Gamma_{q}\left(\sigma-\zeta_{i}+1\right)}+\frac{\left|a_{1}\right|_{i} A_{1} \tau^{\sigma}|\Delta|}{\Gamma_{q}(\sigma+1)}\right. \\
& \left.+\frac{\left(\left|a_{2}\right|_{i} A_{1}+\left|a_{3}\right|_{i} A_{2}\right)|\Delta|}{\Gamma_{q}(\sigma+2)}+\frac{{ }_{i} A_{2}|\Delta|}{\Gamma_{q}(\sigma)}|\Sigma|\right],
\end{aligned}
$$

for $i=1,2, \ldots, m$. Define the operator $\Omega: \mathcal{E} \rightarrow 2^{\mathcal{E}}$ by

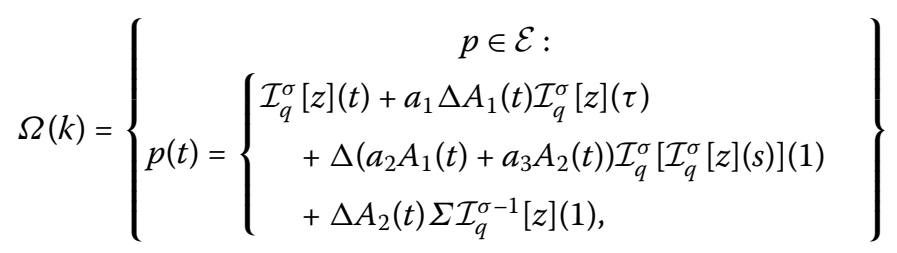

for $z \in S_{\mathcal{T}, k}$. If the multifunction $\Omega$ has the approximate endpoint property, then the boundary value inclusion problem (1)-(2) has a solution. 
Proof First, we prove that $\Omega(k)$ is a closed subset of $\mathcal{P}(\mathcal{E})$ for all $k \in \mathcal{E}$. Since the multivalued map $t \mapsto \mathcal{T}\left(t, k(t), k^{\prime}(t),{ }^{c} \mathcal{D}^{\zeta_{1}} k(t), \ldots,{ }^{c} \mathcal{D}^{\zeta_{m}} k(t)\right)$ is measurable and has closed values for all $k \in \mathcal{E}$, has measurable selection and so $S_{\mathcal{T}, k}$ is nonempty for all $k$. Assume that $k \in \mathcal{E}$ and $\left\{v_{n}\right\}_{n \geq 1}$ be a sequence in $\Omega(k)$ with $v_{n} \rightarrow v$. For every $n \geq 1$, choose $z_{n} \in S_{\mathcal{T}, k}$ such that

$$
\begin{aligned}
v_{n}(t)= & \mathcal{I}_{q}^{\sigma}\left[z_{n}\right](t)+\Delta a_{1} A_{1}(t) \mathcal{I}_{q}^{\sigma}\left[z_{n}\right](\tau)+\Delta\left(a_{2} A_{1}(t)\right. \\
& \left.+a_{3} A_{2}(t)\right) \mathcal{I}_{q}^{\sigma}\left[\mathcal{I}_{q}^{\sigma}\left[z_{n}\right](s)\right](1)+\Delta A_{2}(t) \Sigma \mathcal{I}_{q}^{\sigma-1}\left[z_{n}\right](1)
\end{aligned}
$$

for all $t \in \bar{J}$. By compactness of $\mathcal{T}$, the sequence $\left\{z_{n}\right\}_{n \geq 1}$ has a subsequence which converges to some $z \in L^{1}(\bar{J})$. We denote this subsequence again by $\left\{z_{n}\right\}_{n \geq 1}$. One can easily check that $z \in S_{\mathcal{T}, k}$ and

$$
\begin{aligned}
v_{n}(t) \rightarrow v(t)= & \mathcal{I}_{q}^{\sigma}[z](t)+\Delta a_{1} A_{1}(t) \mathcal{I}_{q}^{\sigma}[z](\tau) \\
& +\Delta\left(a_{2} A_{1}(t)+a_{3} A_{2}(t)\right) \mathcal{I}_{q}^{\sigma}\left[\mathcal{I}_{q}^{\sigma}[z](s)\right](1) \\
& +\Delta A_{2}(t) \Sigma \mathcal{I}_{q}^{\sigma-1}[z](1)
\end{aligned}
$$

for all $t \in \bar{J}$. This shows that $v \in \Omega(k)$ and so $\Omega$ is closed-valued. On the other hand, $\Omega(k)$ is a bounded set for all $k \in \mathcal{E}$ because $\mathcal{T}$ is a compact multivalued map. Finally, we show that $P_{\rho}(\Omega(k), \Omega(l)) \leq \psi(\|k-l\|)$. Let $k, l \in \mathcal{E}$ and $f_{1} \in \Omega(l)$. Choose $z_{1} \in S_{\mathcal{T}, l}$ such that

$$
\begin{aligned}
f_{1}(t)= & \mathcal{I}_{q}^{\sigma}\left[z_{1}\right](t)+\Delta a_{1} A_{1}(t) \mathcal{I}_{q}^{\sigma}\left[z_{1}\right](\tau) \\
& +\Delta\left(a_{2} A_{1}(t)+a_{3} A_{2}(t)\right) \mathcal{I}_{q}^{\sigma}\left[\mathcal{I}_{q}^{\sigma}\left[z_{1}\right](s)\right](1) \\
& +\Delta A_{2}(t) \Sigma \mathcal{I}_{q}^{\sigma-1}\left[z_{1}\right](1)
\end{aligned}
$$

for almost all $t \in \bar{J}$. Since

$$
\begin{aligned}
P_{\rho}\left(\mathcal{T}\left(t, k(t), k^{\prime}(t),{ }^{c} \mathcal{D}^{\zeta_{1}}[k](t), \ldots,{ }^{c} \mathcal{D}^{\zeta_{m}}[k](t)\right)\right. \\
\left.\quad-\mathcal{T}\left(t, l(t), l^{\prime}(t),{ }^{c} \mathcal{D}^{\zeta_{1}}[l](t), \ldots,{ }^{c} \mathcal{D}^{\zeta_{m}}[l](t)\right)\right) \\
\leq \Xi \gamma(t) \psi\left(|k(t)-l(t)|+\left|k^{\prime}(t)-l^{\prime}(t)\right|+\sum_{i=1}^{m}\left|{ }^{c} \mathcal{D}^{\zeta_{1}}[k](t)-{ }^{c} \mathcal{D}^{\zeta_{i}}[l](t)\right|\right)
\end{aligned}
$$

for $t \in \bar{J}$, there exist $v \in \mathcal{T}\left(t, k(t), k^{\prime}(t),{ }^{c} \mathcal{D}^{\zeta_{1}}[k](t), \ldots,{ }^{c} \mathcal{D}^{\zeta_{m}}[k](t)\right)$ such that

$$
\left|z_{1}(t)-v\right| \leq \Xi \gamma(t) \psi\left(|k(t)-l(t)|+\left|k^{\prime}(t)-l^{\prime}(t)\right|+\sum_{i=1}^{m}\left|{ }^{c} \mathcal{D}^{\zeta_{1}}[k](t)-{ }^{c} \mathcal{D}^{\zeta_{1}}[l](t)\right|\right),
$$

for $t \in \bar{J}$. Now, we consider the multivalued map $K: \bar{J} \rightarrow \mathcal{P}(\mathbb{R})$ which is given by

$$
\begin{aligned}
K(t)= & \left\{v \in \mathbb{R}:\left|z_{1}(t)-v\right| \leq \Xi \gamma(t)\right. \\
& \left.\times \psi\left(|k(t)-l(t)|+\left|k^{\prime}(t)-l^{\prime}(t)\right|+\sum_{i=1}^{m}\left|{ }^{c} \mathcal{D}^{\zeta_{1}}[k](t)-{ }^{c} \mathcal{D}^{\zeta_{1}}[l](t)\right|\right)\right\} .
\end{aligned}
$$


Since $z_{1}$ and

$$
\Xi \gamma(t) \psi\left(|k(t)-l(t)|+\left|k^{\prime}(t)-l^{\prime}(t)\right|+\sum_{i=1}^{m}\left|{ }^{c} \mathcal{D}^{\zeta_{1}}[k](t)-{ }^{c} \mathcal{D}^{\zeta_{1}}[l](t)\right|\right),
$$

are measurable, the multifunction

$$
K(\cdot) \cap \mathcal{T}\left(\cdot, k(\cdot), k^{\prime}(\cdot),{ }^{c} \mathcal{D}^{\zeta_{1}}[k](\cdot), \ldots,{ }^{c} \mathcal{D}^{\zeta_{m}}[k](\cdot)\right)
$$

is measurable. Now, choose $z_{2}(t) \in \mathcal{T}\left(t, k(t), k^{\prime}(t),{ }^{c} \mathcal{D}^{\zeta_{1}}[k](t), \ldots,{ }^{c} \mathcal{D}^{\zeta_{m}}[k](t)\right)$ such that

$$
\left|z_{1}(t)-z_{2}(t)\right| \leq \Xi \gamma(t) \psi\left(|k(t)-l(t)|+\left|k^{\prime}(t)-l^{\prime}(t)\right|+\sum_{i=1}^{m}\left|{ }^{c} \mathcal{D}^{\zeta_{1}}[k](t)-{ }^{c} \mathcal{D}^{\zeta_{1}}[l](t)\right|\right),
$$

for all $t \in \bar{J}$. Define the element $f_{2} \in \Omega(k)$ by

$$
\begin{aligned}
f_{2}(t)= & \mathcal{I}_{q}^{\sigma}\left[z_{2}\right](t)+\Delta a_{1} A_{1}(t) \mathcal{I}_{q}^{\sigma}\left[z_{2}\right](\tau) \\
& +\Delta\left(a_{2} A_{1}(t)+a_{3} A_{2}(t)\right) \mathcal{I}_{q}^{\sigma}\left[\mathcal{I}_{q}^{\sigma}\left[z_{2}\right](s)\right](1) \\
& +\Delta A_{2}(t) \Sigma \mathcal{I}_{q}^{\sigma-1}\left[z_{2}\right](1)
\end{aligned}
$$

for all $t \in \bar{J}$. Let $\sup _{t \in \bar{J}}|\gamma(t)|=\|\gamma\|$. Thus, one can get

$$
\begin{aligned}
\left|f_{1}(t)-f_{2}(t)\right| \leq & \mathcal{I}_{q}^{\sigma}\left[\left|z_{1}-z_{2}\right|\right](t)+\Delta a_{1} A_{1}(t) \mathcal{I}_{q}^{\sigma}\left[\left|z_{1}-z_{2}\right|\right](\tau) \\
& +\Delta\left(a_{2} A_{1}(t)+a_{3} A_{2}(t)\right) \mathcal{I}_{q}^{\sigma}\left[\mathcal{I}_{q}^{\sigma}\left[\left|z_{1}-z_{2}\right|\right](s)\right](1) \\
& +\Delta A_{2}(t) \Sigma \mathcal{I}_{q}^{\sigma-1}\left[\left|z_{1}-z_{2}\right|\right](1) \\
\leq & \Xi\|\gamma\| \psi(\|k-l\|)\left[\frac{1}{\Gamma_{q}(\sigma+1)}+\frac{\left|a_{1}\right|}{|\Delta|}{ }_{0} A_{1} \frac{\tau^{\sigma}}{\Gamma_{q}(\sigma+1)}\right. \\
& \left.+\frac{|\Delta|\left(\left|a_{2}\right|_{0} A_{1}+\left|a_{3}\right|_{0} A_{2}\right)}{\Gamma_{q}(\sigma+2)}+\frac{|\Delta|_{0} A_{2}|\Sigma|}{\Gamma_{q}(\sigma)}\right] \\
= & \Lambda_{1} \Xi \psi(\|k-l\|) .
\end{aligned}
$$

On the other hand,

$$
\begin{aligned}
\left|f_{1}^{\prime}(t)-f_{2}^{\prime}(t)\right| \leq & \Xi\|\gamma\| \psi(\|k-l\|)\left[\frac{1}{\Gamma(\sigma-1)}+\left.|\Sigma|\left|a_{1}\right||\Delta|\right|_{0} A_{1}^{\prime} \mid \frac{\tau^{\sigma}}{\Gamma_{q}(\sigma+1)}\right. \\
& \left.+\frac{|\Delta|\left(\left|a_{2}\right|_{0} A_{1}^{\prime}+\left|a_{3}\right|_{0} A_{2}^{\prime}\right)}{\Gamma_{1}(\sigma+2)}+\frac{|\Delta|_{0} A_{2}^{\prime}|\Sigma|}{\Gamma_{q}(\sigma)}\right] \\
= & \Lambda_{2} \Xi \psi(\|k-l\|)
\end{aligned}
$$

and, for each $i=1, \ldots, k$, we have

$$
\begin{aligned}
\left|{ }^{c} \mathcal{D}_{q}^{\zeta_{i}}\left[f_{1}\right](t)-{ }^{c} \mathcal{D}_{q}^{\zeta_{i}}\left[f_{2}\right](t)\right| \leq & \Xi\|\gamma\| \psi(\|k-l\|) \\
& \times\left[\frac{1}{\Gamma_{q}\left(\sigma-\zeta_{i}+1\right)}+\left|a_{1} \| \Delta\right| \frac{{ }_{i} A_{1} \tau^{\sigma}}{\Gamma_{q}(\sigma+1)}\right.
\end{aligned}
$$




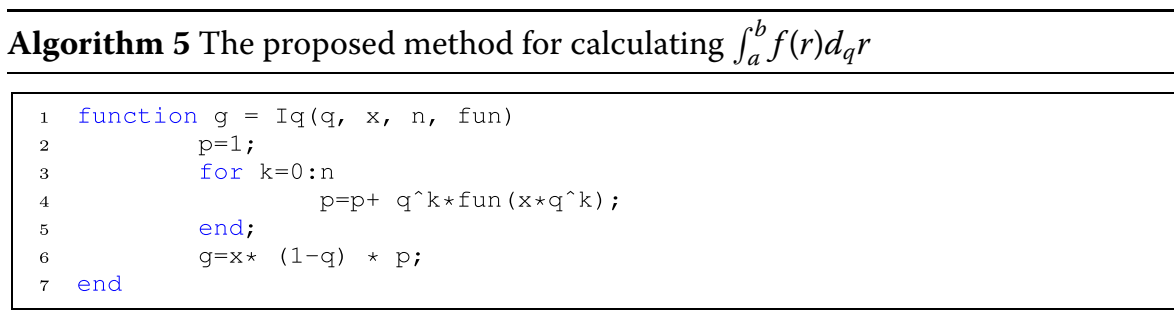

$$
\begin{aligned}
& \left.+\frac{|\Delta|\left(\left|a_{2}\right|_{i} A_{1}+\left|a_{3}\right|_{i} A_{2}\right)}{\Gamma_{q}(\sigma+2)}+\frac{|\Delta|_{i} A_{2}|\Sigma|}{\Gamma_{q}(\sigma)}\right] \\
= & \Xi_{i} \Lambda_{3} \psi(\|k-l\|) .
\end{aligned}
$$

Thus, we get

$$
\begin{aligned}
\left\|f_{1}-f_{2}\right\|= & \sup _{t \in \bar{J}}\left|f_{1}(t)-f_{2}(t)\right|+\sup _{t \in \bar{J}}\left|f_{1}^{\prime}(t)-f_{2}^{\prime}(t)\right| \\
& +\sum_{i=1}^{m} \sup _{t \in \bar{J}}\left|{ }^{c} \mathcal{D}_{q}^{\zeta_{i}}\left[f_{1}\right](t)-{ }^{c} \mathcal{D}_{q}^{\zeta_{i}}\left[f_{2}\right](t)\right| \\
\leq & \Xi \psi(\|k-l\|)(\Xi)=\psi(\|k-l\|) .
\end{aligned}
$$

Hence, $P_{\rho}(\Omega(k), \Omega(l)) \leq \psi(\|k-l\|)$ for all $k, l \in \mathcal{E}$. By using the hypothesis, the multifunction $\Omega$ has approximate endpoint property. Now by using Lemma 1 , there exists $k^{*} \in \mathcal{E}$ such that $\Omega\left(k^{*}\right)=\left\{k^{*}\right\}$. Consequently, $k^{*}$ is a solution for the $q$-inclusion problem (1)(2).

\section{An example by using the algorithms and some numerical calculations}

Here, we give an example to illustrate our main result. In this way, we give a computational technique for checking the problem. We need to present a simplified analysis that is able to execute the values of the $q$-Gamma function. For this purpose, we provided a pseudocode description of the method for calculation of the $q$-Gamma function of order $n$ in the Algorithms 2, 3, 4 and 5. The Algorithm 6 help us for numerical solving of the problem. Also, we provide some figures and numerical tables.

Example 1 Consider the fractional $q$-differential inclusions

$$
\begin{aligned}
{ }^{c} \mathcal{D}_{q}^{\frac{14}{5}}[k](t) \in[ & {\left[0, \frac{3 t \cos ^{2} k(t)}{20\left(1+\cos ^{2} k(t)\right)}+\frac{3 t\left|k^{\prime}(t)\right|}{20\left(1+\left|k^{\prime}(t)\right|\right)}\right.} \\
& +\frac{3 t\left|\sin \left({ }^{c} \mathcal{D}_{q}^{\frac{11}{9}}[k](t)\right)\right|}{20\left(1+\left|\sin \left({ }^{c} \mathcal{D}_{q}^{\frac{14}{5}}[k](t)\right)\right|\right)}+\frac{3 t \exp \left(\left|{ }^{c} \mathcal{D}_{q}^{\frac{8}{5}}[k](t)\right|\right)}{20\left(1+\exp \left(\left|{ }^{c} \mathcal{D}_{q}^{\frac{8}{5}}[k](t)\right|\right)\right)} \\
& \left.+\frac{3 t\left|{ }^{c} \mathcal{D}_{q}^{\frac{7}{6}}[k](t)\right|}{20\left(1+\left|{ }^{c} \mathcal{D}_{q}^{\frac{7}{6}}[k](t)\right|\right)}+\frac{3 t\left({ }^{c} \mathcal{D}_{q}^{\frac{11}{8}}[k](t)\right)^{2}}{20\left(1+\left({ }^{c} \mathcal{D}_{q}^{\frac{11}{8}}[k](t)\right)^{2}\right)}\right]
\end{aligned}
$$


with the sum and integral boundary conditions

$$
\left\{\begin{array}{l}
k(0)+\Sigma k^{\prime \prime}(0)=0, \\
\frac{37}{100} k\left(\frac{11}{80}\right)+\frac{19}{100} \varrho(1)=0, \\
\Sigma k^{\prime}(1)+\frac{9}{100} \varrho(1)=0,
\end{array}\right.
$$

where $t \in[0,1]$ and $\varrho(v)=\int_{0}^{v} \sin (k(r)) \mathrm{d} r$. Consider the set-valued map $\mathcal{T}: \bar{J} \times \mathbb{R}^{6} \rightarrow$ $\mathcal{P}_{c p}(\mathbb{R})$ defined by

$$
\begin{aligned}
\mathcal{T}\left(t, k_{1}, k_{2}, k_{3}, k_{4}, k_{5}, k_{6}\right)= & {\left[0, \frac{3 t \cos ^{2} k_{1}(t)}{20\left(1+\cos ^{2} k_{1}(t)\right)}+\frac{3 t\left|k_{2}(t)\right|}{20\left(1+\left|k_{2}(t)\right|\right)}\right.} \\
& +\frac{3 t\left|\sin k_{3}(t)\right|}{20\left(1+\left|\sin k_{3}(t)\right|\right)}+\frac{3 t e^{\left|k_{4}(t)\right|}}{20\left(1+e^{\left|k_{4}(t)\right|}\right)} \\
& \left.+\frac{3 t\left|k_{5}(t)\right|}{20\left(1+\left|k_{5}(t)\right|\right)}+\frac{3 t\left(k_{6}(t)\right)^{2}}{20\left(1+\left(k_{6}(t)\right)^{2}\right)}\right] .
\end{aligned}
$$

It is clear that $\sigma=\frac{14}{5}, \zeta_{1}=\frac{11}{9}, \zeta_{2}=\frac{8}{5}, \zeta_{3}=\frac{7}{6}, \zeta_{4}=\frac{11}{8}, m=4, a_{1}=\frac{37}{100}, a_{2}=\frac{19}{100}, a_{3}=\frac{9}{100}, \tau=$ $\frac{11}{80}, k=5, \Sigma=\sum_{j=1}^{5} c_{j}=\frac{1}{10}$ with $c_{1}=c_{2}=c_{3}=c_{4}=c_{5}=\frac{1}{50}$. In this case, we have $\gamma:[0,1] \rightarrow$ $[0, \infty)$ by $\gamma(t)=\frac{3}{20} t$ and $\|\gamma\|=\frac{3}{20}$. Put $\psi(t)=\frac{t}{5}$. Clearly, the function $\psi$ is nondecreasing upper semi-continuous on $[0,1]$ such that $\liminf _{t \rightarrow \infty}(t-\psi(t))>0$ and $\psi(t)<t$ for all $t>0$. From (5), (9) and (10), we get

$$
\begin{aligned}
\Delta= & {\left[\left(a_{1} \tau+\frac{a_{2}}{2}\right)\left(2 \Sigma+\frac{a_{3}}{3}-2 a_{3} \Sigma\right)\right.} \\
& \left.+\left(\Sigma+\frac{a_{3}}{2}\right)\left(2 \Sigma\left(a_{1}+a_{2}\right)-\left(a_{1} \tau^{2}+\frac{a_{2}}{3}\right)\right)\right]^{-1} \\
= & {\left[\left(\frac{37}{100} \frac{11}{80}+\frac{19}{200}\right)\left(\frac{1}{5}+\frac{3}{100}-\frac{9}{50} \frac{1}{10}\right)\right.} \\
& \left.+\left(\frac{1}{10}+\frac{9}{200}\right)\left(\frac{14}{125}-\left(\frac{37}{100}\left(\frac{11}{80}\right)^{2}+\frac{19}{300}\right)\right)\right]^{-1}=27.0505
\end{aligned}
$$

and

$$
\begin{aligned}
{ }_{0} A_{1} & =2\left(|\Sigma|+\frac{\left|a_{3}\right|}{2}\right)(1+|\Sigma|)+2\left|a_{3}\right||\Sigma| \\
& =2\left(|0.1|+\frac{9}{200}\right)(1+|0.1|)+\frac{9}{50}|0.1|=0.3370, \\
{ }_{0} A_{1}^{\prime} & =\left(2\left|a_{3}\right|+1\right)|\Sigma|+\frac{\left|a_{3}\right|}{2} \\
& =\left(2 \frac{9}{100}+1\right)|0.1|+\frac{9}{200}=0.1630 \\
{ }_{0} A_{2} & =\left(\left|a_{1}\right| \tau+\frac{\left|a_{2}\right|}{2}\right)(2|\Sigma|+1)+\left|a_{1}\right| \tau^{2}+\frac{\left|a_{2}\right|}{3}+2\left|a_{1}+a_{2}\right||\Sigma| \\
& =1.2\left(\frac{407}{8000}+\frac{19}{200}\right)+\frac{37}{100}\left(\frac{11}{80}\right)^{2}+\frac{19}{300}+\frac{28}{25}|0.1|=0.3574,
\end{aligned}
$$


Table 1 Some numerical results for ${ }_{1} A_{1}$ and ${ }_{1} A_{2}$ in Example 1 for $q=\frac{1}{10}, \frac{1}{2}, \frac{6}{7}$

\begin{tabular}{|c|c|c|c|c|c|c|}
\hline \multirow[b]{2}{*}{$n$} & \multicolumn{2}{|l|}{$q=\frac{1}{10}$} & \multicolumn{2}{|l|}{$q=\frac{1}{2}$} & \multicolumn{2}{|l|}{$q=\frac{6}{7}$} \\
\hline & ${ }_{1} A_{1}$ & ${ }_{1} A_{2}$ & ${ }_{1} A_{1}$ & ${ }_{1} A_{2}$ & ${ }_{1} A_{1}$ & ${ }_{1} A_{2}$ \\
\hline 1 & 0.4478 & 0.4678 & 0.4226 & 0.442 & 0.3201 & 0.3423 \\
\hline 2 & 0.4479 & 0.4679 & 0.4353 & 0.4544 & 0.3365 & 0.3575 \\
\hline 3 & $\underline{0.448}$ & 0.4679 & 0.4417 & 0.4606 & 0.3518 & 0.3722 \\
\hline 4 & 0.448 & 0.4679 & 0.4449 & 0.4638 & 0.3654 & 0.3853 \\
\hline 5 & 0.448 & 0.4679 & 0.4465 & 0.4654 & 0.3773 & 0.3969 \\
\hline & & : & $:$ & . & : & : \\
\hline 14 & 0.448 & 0.4679 & 0.4481 & 0.4669 & 0.4316 & 0.4501 \\
\hline 15 & 0.448 & 0.4679 & $\underline{0.4482}$ & $\underline{0.4669}$ & 0.4342 & 0.4526 \\
\hline 16 & 0.448 & 0.4679 & 0.4482 & 0.4669 & 0.4364 & 0.4548 \\
\hline 17 & 0.448 & 0.4679 & 0.4482 & 0.4669 & 0.4383 & 0.4567 \\
\hline : & $\vdots$ & $\vdots$ & $\vdots$ & $\vdots$ & $\vdots$ & $\vdots$ \\
\hline 49 & 0.448 & 0.4679 & 0.4482 & 0.4669 & 0.4496 & 0.4678 \\
\hline 50 & 0.448 & 0.4679 & 0.4482 & 0.4669 & $\underline{0.4496}$ & $\underline{0.4679}$ \\
\hline 51 & 0.448 & 0.4679 & 0.4482 & 0.4669 & 0.4496 & 0.4679 \\
\hline 52 & 0.448 & 0.4679 & 0.4482 & 0.4669 & 0.4496 & 0.4679 \\
\hline
\end{tabular}

$$
\begin{aligned}
{ }_{0} A_{2}^{\prime}= & 2\left(\left|a_{1}\right| \tau+\frac{\left|a_{2}\right|}{2}\right)+\left|a_{1}\right| \tau^{2}+\frac{\left|a_{2}\right|}{3}+2\left|a_{1}+a_{2}\right||\Sigma| \\
= & 2\left(\frac{407}{8000}+\frac{19}{200}\right)+\frac{37}{100}\left(\frac{11}{80}\right)^{2}+\frac{19}{300}+\frac{28}{25}|0.1|=0.4741, \\
{ }_{i} A_{1}= & \left(\Sigma+\frac{\left|a_{3}\right|}{2}\right)\left[\frac{1}{\Gamma_{q}\left(2-\zeta_{i}\right)}+\frac{2}{\Gamma\left(3-\zeta_{i}\right)}\right]+2\left|a_{3}\right||\Sigma| \frac{1}{\Gamma_{q}\left(2-\zeta_{i}\right)} \\
= & \left(0.1+\frac{9}{200}\right)\left[\frac{1}{\Gamma_{q}\left(2-\zeta_{i}\right)}+\frac{2}{\Gamma_{q}\left(3-\zeta_{i}\right)}\right]+\frac{0.18|0.1|}{\Gamma_{q}\left(2-\zeta_{i}\right)}, \\
{ }_{i} A_{2}= & \left(\left|a_{1}\right| \tau+\frac{\left|a_{2}\right|}{2}\right) \frac{2}{\Gamma_{q}\left(3-\zeta_{i}\right)} \\
& +\left(\left|a_{1}\right| \tau^{2}+\frac{\left|a_{2}\right|}{3}+2\left|a_{1}+a_{2}\right||\Sigma|\right) \frac{1}{\Gamma_{q}\left(2-\zeta_{i}\right)} \\
= & \left(\frac{407}{8000}+\frac{19}{200}\right) \frac{2}{\Gamma_{q}\left(3-\zeta_{i}\right)} \\
& +\left(\frac{37}{100}\left(\frac{11}{80}\right)^{2}+\frac{19}{300}+\frac{28}{25}|0.1|\right) \frac{1}{\Gamma_{q}\left(2-\zeta_{i}\right)} .
\end{aligned}
$$

Tables 1, 2, 3, 4 show that

$$
\begin{array}{ll}
{ }_{1} A_{1} \approx 0.4480,0.4482,0.4496, & { }_{1} A_{2} \approx 0.4679,0.4669,0.4679, \\
{ }_{2} A_{1} \approx 0.4109,0.4260,0.4001, & { }_{2} A_{2} \approx 0.4260,0.4128,0.4111, \\
{ }_{3} A_{1} \approx 0.4500,0.4508,0.4521, & { }_{3} A_{2} \approx 0.4704,0.4703,0.4712, \\
{ }_{4} A_{1} \approx 0.4386,0.4354,0.4364, & { }_{4} A_{2} \approx 0.4572,0.4519,0.4521,
\end{array}
$$

for $q=\frac{1}{10}, \frac{1}{2}, \frac{6}{7}$, respectively. By using (8), we get

$$
M_{1}=\|\gamma\|\left[\frac{1}{\Gamma_{q}(\sigma+1)}+\frac{\left|a_{1}\right|_{0} A_{1} \tau^{\sigma}|\Delta|}{\Gamma_{q}(\sigma+1)}\right.
$$


Table 2 Some numerical results for ${ }_{2} A_{1}$ and ${ }_{2} A_{2}$ in Example 1 for $q=\frac{1}{10}, \frac{1}{2}, \frac{6}{7}$

\begin{tabular}{|c|c|c|c|c|c|c|}
\hline \multirow[b]{2}{*}{$n$} & \multicolumn{2}{|l|}{$q=\frac{1}{10}$} & \multicolumn{2}{|l|}{$q=\frac{1}{2}$} & \multicolumn{2}{|l|}{$q=\frac{6}{7}$} \\
\hline & ${ }_{2} A_{1}$ & ${ }_{2} A_{2}$ & ${ }_{2} A_{1}$ & ${ }_{2} A_{2}$ & ${ }_{2} A_{1}$ & ${ }_{2} A_{2}$ \\
\hline 1 & 0.4111 & 0.4262 & 0.3941 & 0.4076 & 0.3713 & 0.3916 \\
\hline 2 & $\underline{0.4109}$ & $\underline{0.426}$ & 0.397 & 0.4097 & 0.3663 & 0.3836 \\
\hline 3 & $\overline{0.4109}$ & $\overline{0.426}$ & 0.3988 & 0.4112 & 0.3674 & 0.383 \\
\hline & & & & : & : & : \\
\hline 8 & 0.4109 & 0.426 & 0.4007 & 0.4127 & 0.3827 & 0.3951 \\
\hline 9 & 0.4109 & 0.426 & $\underline{0.426}$ & $\underline{0.4128}$ & 0.3851 & 0.3972 \\
\hline 10 & 0.4109 & 0.426 & $\overline{0.4007}$ & $\overline{0.4128}$ & 0.3871 & 0.3991 \\
\hline 11 & 0.4109 & 0.426 & 0.4007 & 0.4128 & 0.389 & 0.4008 \\
\hline & $\vdots$ & $\vdots$ & $\vdots$ & $\vdots$ & $\vdots$ & $\vdots$ \\
\hline 43 & 0.4109 & 0.426 & 0.4007 & 0.4128 & 0.4 & 0.411 \\
\hline 44 & 0.4109 & 0.426 & 0.4007 & 0.4128 & 0.4001 & 0.411 \\
\hline 45 & 0.4109 & 0.426 & 0.4007 & 0.4128 & 0.4001 & $\underline{0.4111}$ \\
\hline 46 & 0.4109 & 0.426 & 0.4007 & 0.4128 & $\overline{0.4001}$ & $\overline{0.4111}$ \\
\hline 47 & 0.4109 & 0.426 & 0.4007 & 0.4128 & 0.4001 & 0.4111 \\
\hline
\end{tabular}

$$
\begin{aligned}
& \left.+\frac{\left(\left|a_{2}\right|_{0} A_{1}+\left|a_{3}\right|_{0} A_{2}\right)|\Delta|}{\Gamma_{q}(\sigma+2)}+\frac{{ }_{0} A_{2}|\Delta|}{\Gamma_{q}(\sigma)}|\Sigma|\right], \\
= & \frac{3}{20}\left[\frac{1}{\Gamma_{q}\left(\frac{14}{5}+1\right)}+\frac{\frac{37 \times 0.3370}{100}\left(\frac{11}{80}\right) \frac{14}{5} 27.0505}{\Gamma_{q}\left(\frac{14}{5}+1\right)}\right. \\
& \left.+\frac{\left(\frac{19 \times 0.3370}{100}+\frac{9 \times * 0.3574}{100}\right) 27.0505}{\Gamma_{q}\left(\frac{14}{5}+2\right)}+\frac{0.3574 \times|0.1|}{\Gamma_{q}\left(\frac{14}{5}\right)}|27.0505|\right], \\
M_{2}= & \|\gamma\|\left[\frac{1}{\Gamma_{q}(\sigma-1)}+\frac{\left|a_{1}\right|_{0} A_{1}^{\prime} \tau^{\sigma}|\Delta|}{\Gamma_{q}(\sigma+1)}\right. \\
& \left.+\frac{\left(\left|a_{2}\right|_{0} A_{1}^{\prime}+\left|a_{3}\right|_{0} A_{2}^{\prime}\right)|\Delta|}{\Gamma_{q}(\sigma+2)}+\frac{{ }_{0} A_{2}^{\prime}|\Delta|}{\Gamma_{q}(\sigma)}|\Sigma|\right] \\
= & \frac{3}{20}\left[\frac{1}{\Gamma_{q}\left(\frac{14}{5}-1\right)}+\frac{\frac{37 \times 0.1630}{100}\left(\frac{11}{80}\right) \frac{15}{4} 27.0505}{\Gamma_{q}\left(\frac{15}{4}+1\right)}\right. \\
& \left.+\frac{\left(\frac{19 \times 0.1630}{100}+\frac{9 \times 0.4741}{100}\right)|27.0505|}{\Gamma_{q}\left(\frac{15}{4}+2\right)}+\frac{0.4741|27.0505|}{\Gamma_{q}\left(\frac{15}{4}\right)}|0.1|\right] .
\end{aligned}
$$

Table 5 shows that $M_{1} \approx 0.5015,0.1483,0.0617$ and $M_{2} \approx 0.5053,0.2350,0.1570$ for $q=$ $\frac{1}{10}, \frac{1}{2}, \frac{6}{7}$, respectively (Fig. 1 ). By using (10), we obtain

$$
\begin{aligned}
{ }_{i} M_{3}= & \|\gamma\|\left[\frac{1}{\Gamma_{q}\left(\sigma-\zeta_{i}+1\right)}+\frac{\left|a_{1}\right|{ }_{i} A_{1} \tau^{\sigma}|\Delta|}{\Gamma_{q}(\sigma+1)}\right. \\
& \left.+\frac{\left(\left|a_{2}\right| A_{i} A_{1}+\left|a_{3}\right|{ }_{i} A_{2}\right)|\Delta|}{\Gamma_{q}(\sigma+2)}+\frac{{ }_{i} A_{2}|\Delta|}{\Gamma_{q}(\sigma)}|\Sigma|\right] \\
= & \frac{3}{20}\left[\frac{1}{\Gamma_{q}\left(\frac{14}{5}-\zeta_{i}+1\right)}+\frac{\frac{37}{100} i A_{1}\left(\frac{11}{80}\right)^{\frac{14}{5}} 27.0505}{\Gamma_{q}\left(\frac{14}{5}+1\right)}\right. \\
& \left.+\frac{\left(\frac{19}{100} i A_{1}+\frac{9}{100} i A_{2}\right) 27.0505}{\Gamma_{q}\left(\frac{14}{5}+2\right)}+\frac{27.0505_{i} A_{2}}{\Gamma_{q}\left(\frac{14}{5}\right)}|0.1|\right] .
\end{aligned}
$$


Table 3 Some numerical results for ${ }_{3} A_{1}$ and ${ }_{3} A_{2}$ in Example 1 for $q=\frac{1}{10}, \frac{1}{2}, \frac{6}{7}$

\begin{tabular}{|c|c|c|c|c|c|c|}
\hline \multirow[b]{2}{*}{$n$} & \multicolumn{2}{|l|}{$q=\frac{1}{10}$} & \multicolumn{2}{|l|}{$q=\frac{1}{2}$} & \multicolumn{2}{|l|}{$q=\frac{6}{7}$} \\
\hline & ${ }_{3} A_{1}$ & ${ }_{3} A_{2}$ & ${ }_{3} A_{1}$ & ${ }_{3} A_{2}$ & ${ }_{3} A_{1}$ & ${ }_{3} A_{2}$ \\
\hline 1 & 0.4498 & 0.4702 & 0.4224 & 0.4423 & 0.3082 & 0.33 \\
\hline 2 & 0.45 & 0.4703 & 0.4366 & 0.4562 & 0.3274 & 0.3484 \\
\hline 3 & 0.45 & 0.4704 & 0.4437 & 0.4632 & 0.3447 & 0.3652 \\
\hline 4 & 0.45 & 0.4704 & 0.4472 & 0.4667 & 0.3599 & 0.38 \\
\hline . & $\vdots$ & $\vdots$ & $\vdots$ & $\vdots$ & $\vdots$ & $\vdots$ \\
\hline 11 & 0.45 & 0.4704 & 0.4508 & 0.4702 & 0.4208 & 0.4401 \\
\hline 12 & 0.45 & 0.4704 & $\underline{0.4508}$ & $\underline{0.4703}$ & 0.4253 & 0.4446 \\
\hline 13 & 0.45 & 0.4704 & $\overline{0.4508}$ & $\overline{0.4703}$ & 0.4291 & 0.4484 \\
\hline 14 & 0.45 & 0.4704 & 0.4508 & 0.4703 & 0.4324 & 0.4517 \\
\hline & $\vdots$ & $\vdots$ & & $\vdots$ & $\vdots$ & \\
\hline 47 & 0.45 & 0.4704 & 0.4508 & 0.4703 & 0.452 & 0.4711 \\
\hline 48 & 0.45 & 0.4704 & 0.4508 & 0.4703 & 0.4521 & 0.4712 \\
\hline 49 & 0.45 & 0.4704 & 0.4508 & 0.4703 & 0.4521 & 0.4712 \\
\hline 50 & 0.45 & 0.4704 & 0.4508 & 0.4703 & 0.4521 & 0.4712 \\
\hline
\end{tabular}

Table 4 Some numerical results for ${ }_{4} A_{1}$ and ${ }_{4} A_{2}$ in Example 1 for $q=\frac{1}{10}, \frac{1}{2}, \frac{6}{7}$

\begin{tabular}{|c|c|c|c|c|c|c|}
\hline \multirow[b]{2}{*}{$n$} & \multicolumn{2}{|l|}{$q=\frac{1}{10}$} & \multicolumn{2}{|l|}{$q=\frac{1}{2}$} & \multicolumn{2}{|l|}{$q=\frac{6}{7}$} \\
\hline & ${ }_{4} A_{1}$ & ${ }_{4} A_{2}$ & ${ }_{4} A_{1}$ & ${ }_{4} A_{2}$ & ${ }_{4} A_{1}$ & ${ }_{4} A_{2}$ \\
\hline 1 & 0.4385 & 0.4571 & 0.4179 & 0.4355 & 0.3488 & 0.3713 \\
\hline 2 & 0.4386 & 0.4572 & 0.4265 & 0.4435 & 0.3565 & 0.3771 \\
\hline 3 & $\overline{0.4386}$ & $\overline{0.4572}$ & 0.4309 & 0.4477 & 0.366 & 0.3853 \\
\hline & : & : & : & : & : & : \\
\hline 7 & 0.4386 & 0.4572 & 0.4352 & 0.4517 & 0.3972 & 0.4143 \\
\hline 8 & 0.4386 & 0.4572 & 0.4353 & 0.4518 & 0.4028 & 0.4196 \\
\hline 9 & 0.4386 & 0.4572 & $\underline{0.4354}$ & $\underline{0.4519}$ & 0.4075 & 0.4242 \\
\hline & $\vdots$ & $\vdots$ & $\vdots$ & $\vdots$ & $\vdots$ & : \\
\hline 44 & 0.4386 & 0.4572 & 0.4354 & 0.4519 & 0.4364 & 0.452 \\
\hline 45 & 0.4386 & 0.4572 & 0.4354 & 0.4519 & 0.4364 & $\underline{0.4521}$ \\
\hline 46 & 0.4386 & 0.4572 & 0.4354 & 0.4519 & $\overline{0.4364}$ & 0.4521 \\
\hline 47 & 0.4386 & 0.4572 & 0.4354 & 0.4519 & 0.4364 & 0.4521 \\
\hline
\end{tabular}

Table 5 Some numerical results for $M_{1}$ and $M_{2}$ in Example 1 for $q=\frac{1}{10}, \frac{1}{2}, \frac{6}{7}$

\begin{tabular}{|c|c|c|c|c|c|c|}
\hline \multirow[b]{2}{*}{$n$} & \multicolumn{2}{|l|}{$q=\frac{1}{10}$} & \multicolumn{2}{|l|}{$q=\frac{1}{2}$} & \multicolumn{2}{|l|}{$q=\frac{6}{7}$} \\
\hline & $\overline{M_{1}}$ & $M_{2}$ & $\overline{M_{1}}$ & $M_{2}$ & $\overline{M_{1}}$ & $M_{2}$ \\
\hline 1 & 0.501 & 0.5047 & 0.117 & 0.1905 & 0.0053 & 0.0257 \\
\hline 2 & 0.5015 & 0.5052 & 0.1321 & 0.2121 & 0.0087 & 0.0372 \\
\hline 3 & 0.5015 & 0.5052 & 0.1401 & 0.2234 & 0.0125 & 0.0488 \\
\hline 4 & $\underline{0.5015}$ & $\underline{0.5053}$ & 0.1442 & 0.2291 & 0.0166 & 0.0601 \\
\hline 5 & 0.5015 & 0.5053 & 0.1463 & 0.232 & 0.0208 & 0.0708 \\
\hline 6 & 0.5015 & 0.5053 & 0.1473 & 0.2335 & 0.0248 & 0.0808 \\
\hline : & & : & : & : & : & $\vdots$ \\
\hline 13 & 0.5015 & 0.5053 & 0.1483 & 0.2349 & 0.0465 & 0.1278 \\
\hline 14 & 0.5015 & 0.5053 & 0.1483 & 0.235 & 0.0485 & 0.1318 \\
\hline 15 & 0.5015 & 0.5053 & $\underline{0.1483}$ & $\underline{0.235}$ & 0.0503 & 0.1352 \\
\hline 16 & 0.5015 & 0.5053 & 0.1483 & $\overline{0.235}$ & 0.0518 & 0.1382 \\
\hline 17 & 0.5015 & 0.5053 & 0.1483 & 0.235 & 0.0532 & 0.1408 \\
\hline 18 & 0.5015 & 0.5053 & 0.1483 & 0.235 & 0.0543 & 0.1431 \\
\hline & $\vdots$ & $\vdots$ & & & & \\
\hline 48 & 0.5015 & 0.5053 & 0.1483 & 0.235 & 0.0617 & 0.1569 \\
\hline 49 & 0.5015 & 0.5053 & 0.1483 & 0.235 & $\underline{0.0617}$ & $\underline{0.157}$ \\
\hline 50 & 0.5015 & 0.5053 & 0.1483 & 0.235 & 0.0617 & $\overline{0.157}$ \\
\hline 51 & 0.5015 & 0.5053 & 0.1483 & 0.235 & 0.0617 & 0.157 \\
\hline
\end{tabular}



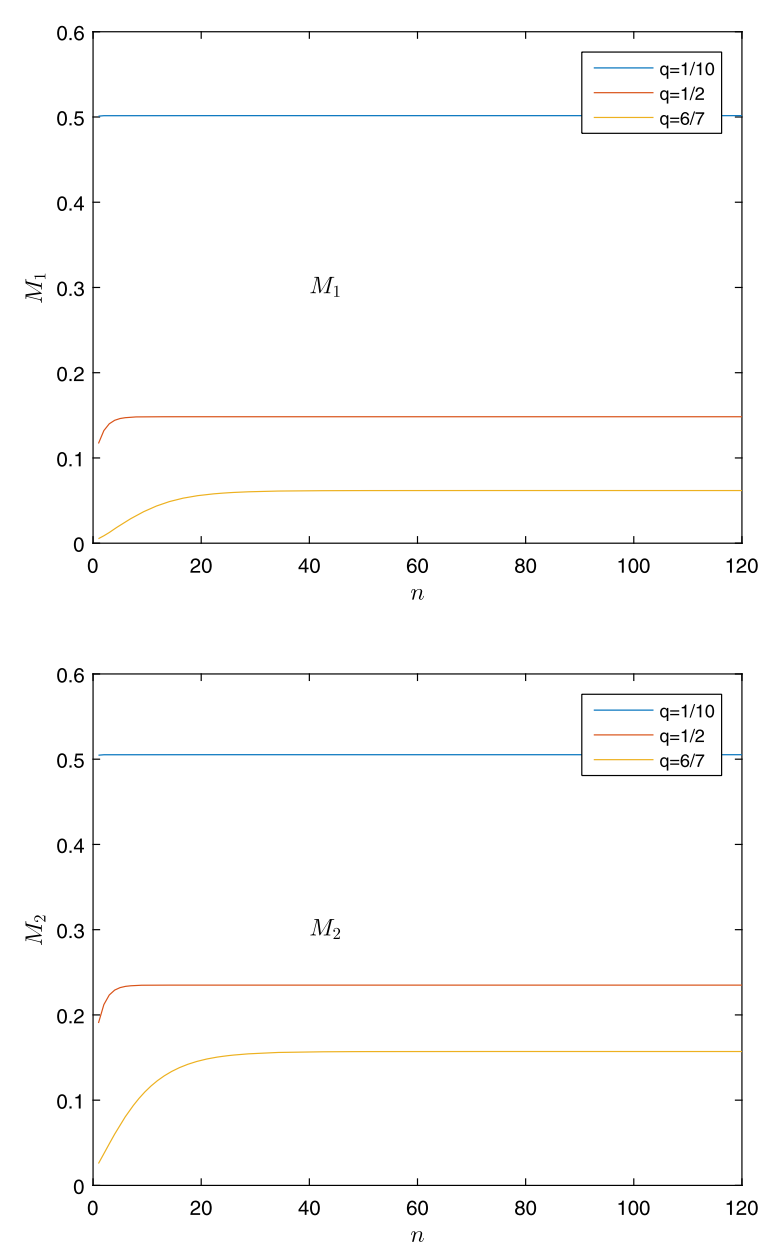

Figure 1 Numerical results of $M_{1}$ and $M_{2}$ where $q=\frac{1}{10}, \frac{1}{2}, \frac{6}{7}$ in Example 1

Table 6 shows that ${ }_{1} M_{3} \approx 0.6407,0.2231,0.1142,{ }_{2} M_{3} \approx 0.6016,0.2237,0.1273,{ }_{3} M_{3} \approx$ $0.6424,0.2218,0.1120$ and ${ }_{4} M_{3} \approx 0.6315,0.2252,0.1199$ for $q=\frac{1}{10}, \frac{1}{2}, \frac{6}{7}$, respectively (Fig. 2). Thus, we can easily get

$$
P_{\rho}\left(\mathcal{T}\left(t, k_{1}, k_{2}, k_{3}, k_{4}, k_{5}, k_{6}\right), \mathcal{T}\left(t, k_{1}^{\prime}, k_{2}^{\prime}, k_{3}^{\prime}, k_{4}^{\prime}, k_{5}^{\prime}, k_{6}^{\prime}\right)\right) \leq \Xi \gamma(t) \psi\left(\sum_{i=1}^{6}\left|k_{i}-k_{i}^{\prime}\right|\right),
$$

for all $k_{i}, k_{i}^{\prime} \in \mathbb{R}(i=1,2,3,4,5,6)$, where

$$
\Xi=\left[M_{1}+M_{2}+{ }_{1} M_{3}+{ }_{2} M_{3}+{ }_{3} M_{3}+{ }_{4} M_{3}\right]^{-1} .
$$

Table 7 shows that $\Xi \approx 0.2839,0.3449,0.3657$ for $q=\frac{1}{10}, \frac{1}{2}, \frac{6}{7}$, respectively (Fig. 3 ). Let $E_{1}=\left\{k: k, k^{\prime},{ }^{c} \mathcal{D}_{q}^{\frac{11}{9}}[k](t) \in \mathcal{A}\right\}, E_{2}=\left\{k: k, k^{\prime},{ }^{c} \mathcal{D}_{q}^{\frac{8}{5}}[k](t) \in \mathcal{A}\right\}, E_{3}=\left\{k: k, k^{\prime},{ }^{c} \mathcal{D}_{q}^{\frac{7}{6}}[k](t) \in \mathcal{A}\right\}$, $E_{4}=\left\{k: k, k^{\prime},{ }^{c} \mathcal{D}_{q}^{\frac{11}{8}}[k](t), \in \mathcal{A}\right\}$ and $\mathcal{E}=E_{1} \times E_{2} \times E_{3} \times E_{4}$. Now, define the operator $\Omega$ : $\mathcal{E} \rightarrow 2^{\mathcal{E}}$ by $\Omega(u)=\left\{p \in \mathcal{E}:\right.$ there exists $v \in S_{\mathcal{T}, k}$ such that $p(t)=v(t)$ for all $\left.t \in \bar{J}\right\}$, where

$$
v(t)=\mathcal{I}_{q}^{\sigma}[z](t)+\Delta a_{1} A_{1}(t) \mathcal{I}_{q}^{\sigma}[z](\tau)
$$


Table 6 Some numerical results of ${ }_{i} M_{3}(i=1,2,3,4)$ in Example 1 for $q=\frac{1}{10}, \frac{1}{2}, \frac{6}{7}$

\begin{tabular}{|c|c|c|c|c|}
\hline$n$ & ${ }_{1} M_{3}$ & $2 M_{3}$ & ${ }_{3} M_{3}$ & ${ }_{4} M_{3}$ \\
\hline 1 & $\begin{array}{l}q=\frac{1}{10} \\
0.6398\end{array}$ & 0.6012 & 0.6415 & 0.6307 \\
\hline 2 & 0.6406 & 0.6016 & 0.6423 & 0.6314 \\
\hline 3 & $\underline{0.6407}$ & 0.6016 & $\underline{0.6424}$ & 0.6314 \\
\hline 4 & $\overline{0.6407}$ & 0.6016 & $\overline{0.6424}$ & 0.6315 \\
\hline 5 & 0.6407 & 0.6016 & 0.6424 & 0.6315 \\
\hline 6 & 0.6407 & 0.6016 & 0.6424 & 0.6315 \\
\hline & \multicolumn{4}{|c|}{$q=\frac{1}{2}$} \\
\hline 1 & 0.171 & 0.1776 & 0.169 & 0.1752 \\
\hline 2 & 0.1959 & 0.1997 & 0.1942 & 0.1991 \\
\hline \multirow[t]{2}{*}{3} & 0.2092 & 0.2115 & 0.2077 & 0.2119 \\
\hline & & $\vdots$ & $\vdots$ & $\vdots$ \\
\hline 10 & 0.223 & 0.2236 & 0.2217 & 0.2251 \\
\hline 11 & 0.2231 & 0.2236 & 0.2217 & 0.2252 \\
\hline 12 & $\overline{0.2231}$ & 0.2236 & $\underline{0.2218}$ & 0.2252 \\
\hline 13 & 0.2231 & $\underline{0.2237}$ & $\overline{0.2218}$ & 0.2252 \\
\hline 14 & 0.2231 & $\overline{0.2237}$ & 0.2218 & 0.2252 \\
\hline 15 & 0.2231 & 0.2237 & 0.2218 & 0.2252 \\
\hline & \multicolumn{4}{|c|}{$q=\frac{6}{7}$} \\
\hline 1 & 0.0104 & 0.0159 & 0.0097 & 0.0124 \\
\hline 2 & 0.0167 & 0.024 & 0.0158 & 0.0195 \\
\hline \multirow[t]{2}{*}{3} & 0.0238 & 0.0327 & 0.0227 & 0.0272 \\
\hline & & $\vdots$ & $\vdots$ & \\
\hline 46 & 0.1141 & 0.1272 & 0.1119 & 0.1198 \\
\hline 47 & 0.1141 & 0.1272 & 0.1119 & 0.1198 \\
\hline 48 & 0.1141 & $\underline{0.1273}$ & 0.1119 & 0.1198 \\
\hline 49 & 0.1141 & $\overline{0.1273}$ & 0.1119 & 0.1199 \\
\hline 50 & 0.1142 & 0.1273 & 0.1119 & 0.1199 \\
\hline 51 & $\overline{0.1142}$ & 0.1273 & 0.112 & 0.1199 \\
\hline 52 & 0.1142 & 0.1273 & $\underline{0.112}$ & 0.1199 \\
\hline 53 & 0.1142 & 0.1273 & $\overline{0.112}$ & 0.1199 \\
\hline 54 & 0.1142 & 0.1273 & 0.112 & 0.1199 \\
\hline
\end{tabular}

$$
\begin{aligned}
& +\Delta\left(a_{2} A_{1}(t)+a_{3} A_{2}(t)\right) \mathcal{I}_{q}^{\sigma}\left[\mathcal{I}_{q}^{\sigma}[z](s)\right](1)+\Delta A_{2}(t) \Sigma \mathcal{I}_{q}^{\sigma-1}[z](1) \\
= & \mathcal{I}_{q}^{\frac{14}{5}}[z](t)+10.0087 A_{1}(t) \mathcal{I}_{q}^{\frac{14}{5}}[z]\left(\frac{11}{80}\right) \\
& +\left(5.1396 A_{1}(t)+2.4345 A_{2}(t)\right) \mathcal{I}_{q}^{\frac{14}{5}}\left[\mathcal{I}_{q}^{\frac{14}{5}}[z](s)\right](1) \\
& +2.70505 A_{2}(t) \mathcal{I}_{q}^{\frac{9}{5}}[z](1)
\end{aligned}
$$

Here, $\mathcal{I}_{q}^{\frac{14}{5}}\left[\mathcal{I}_{q}^{\frac{14}{5}}[z](s)\right](1)=\int_{0}^{1} \int_{0}^{r} \frac{(r-q s)^{\left(\frac{14}{5}-1\right)}}{\Gamma_{q}\left(\frac{14}{5}\right)} z(s) \mathrm{d}_{q} s \mathrm{~d}_{q} r$

$$
\begin{aligned}
A_{1}(t) & =\left(\Sigma+\frac{a_{3}}{2}\right)\left(t^{2}-t-2 \Sigma\right)+2 a_{3} t \Sigma=0.145 t^{2}-0.127 t+0.029 \\
A_{2}(t) & =\left(a_{1} \tau+\frac{a_{2}}{2}\right)\left(2 \Sigma-t^{2}\right)+t\left(a_{1} \tau^{2}+\frac{a_{2}}{3}-2\left(a_{1}+a_{2}\right) \Sigma\right) \\
& =-0.145875 t^{2}+0.041671 t+0.029175
\end{aligned}
$$

The Table 7 shows the values of $\Xi \approx 0.2839,0.3449,0.3657$ for $q=\frac{1}{10}, \frac{1}{2}, \frac{6}{7}$. One can easily check that $\inf _{k \in \mathcal{E}} \sup _{l \in \Omega(k)}\|k-l\|=0$. Thus, the operator $\Omega$ has the approximate 

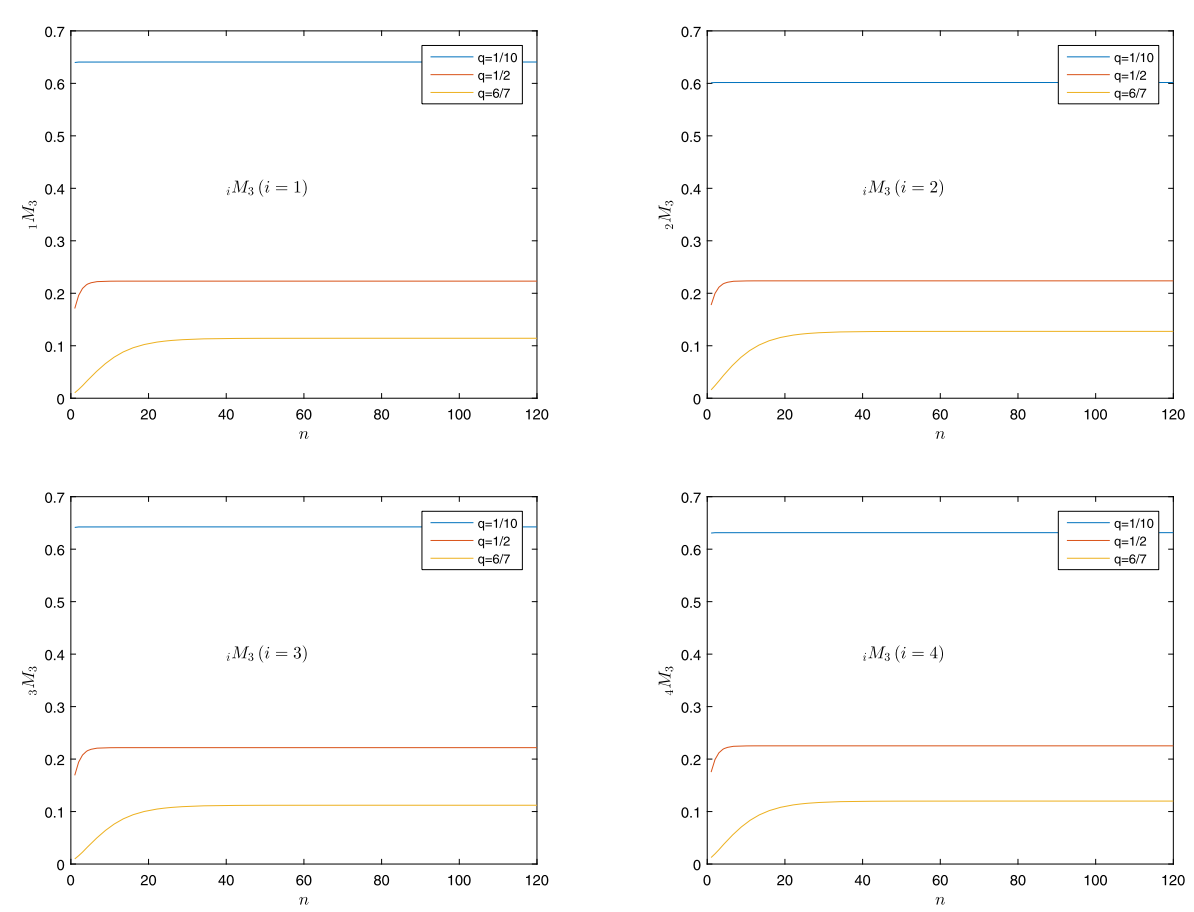

Figure 2 Numerical results of $M_{3},(i=1,2,3,4)$ where $q=\frac{1}{10}, \frac{1}{2}, \frac{6}{7}$ in Example 1

Table 7 Numerical results of $\boldsymbol{\Xi}$ in Example 1 for $q=\frac{1}{10}, \frac{1}{2}, \frac{6}{7}$

\begin{tabular}{clll}
\hline$n$ & $q=\frac{1}{10}$ & $q=\frac{1}{2}$ & $q=\frac{6}{7}$ \\
\hline 1 & 0.2842 & 0.3545 & 0.393 \\
2 & $\frac{0.2839}{0.2839}$ & 0.3496 & 0.3904 \\
3 & $\vdots$ & 0.3473 & 0.388 \\
$\vdots$ & 0.2839 & $\vdots$ & $\vdots$ \\
8 & 0.2839 & 0.345 & 0.3778 \\
9 & 0.2839 & $\underline{0.3449}$ & 0.3763 \\
10 & 0.2839 & 0.3449 & 0.3749 \\
11 & $\vdots$ & 0.3449 & 0.3737 \\
$\vdots$ & 0.2839 & $\vdots$ & $\vdots$ \\
38 & 0.2839 & 0.3449 & 0.3658 \\
39 & 0.2839 & 0.3449 & 0.3657 \\
40 & 0.2839 & 0.3449 & 0.3657 \\
41 & & 0.3449 & 0.3657 \\
\hline
\end{tabular}

endpoint property. Now, by using Theorem 4 , we get the fractional $q$-differential inclusion problem (11) has a solution.

\section{Conclusion}

Most natural phenomena could be modeled by different types of fractional differential equations and inclusions. Recently some physicists have been studying the role of fractional calculus in better describing of physical phenomena. They have found that by using the q-fractional they can provide a better description by some physical notions. Thus, we should investigate distinct fractional differential equations and inclusions to increase our 


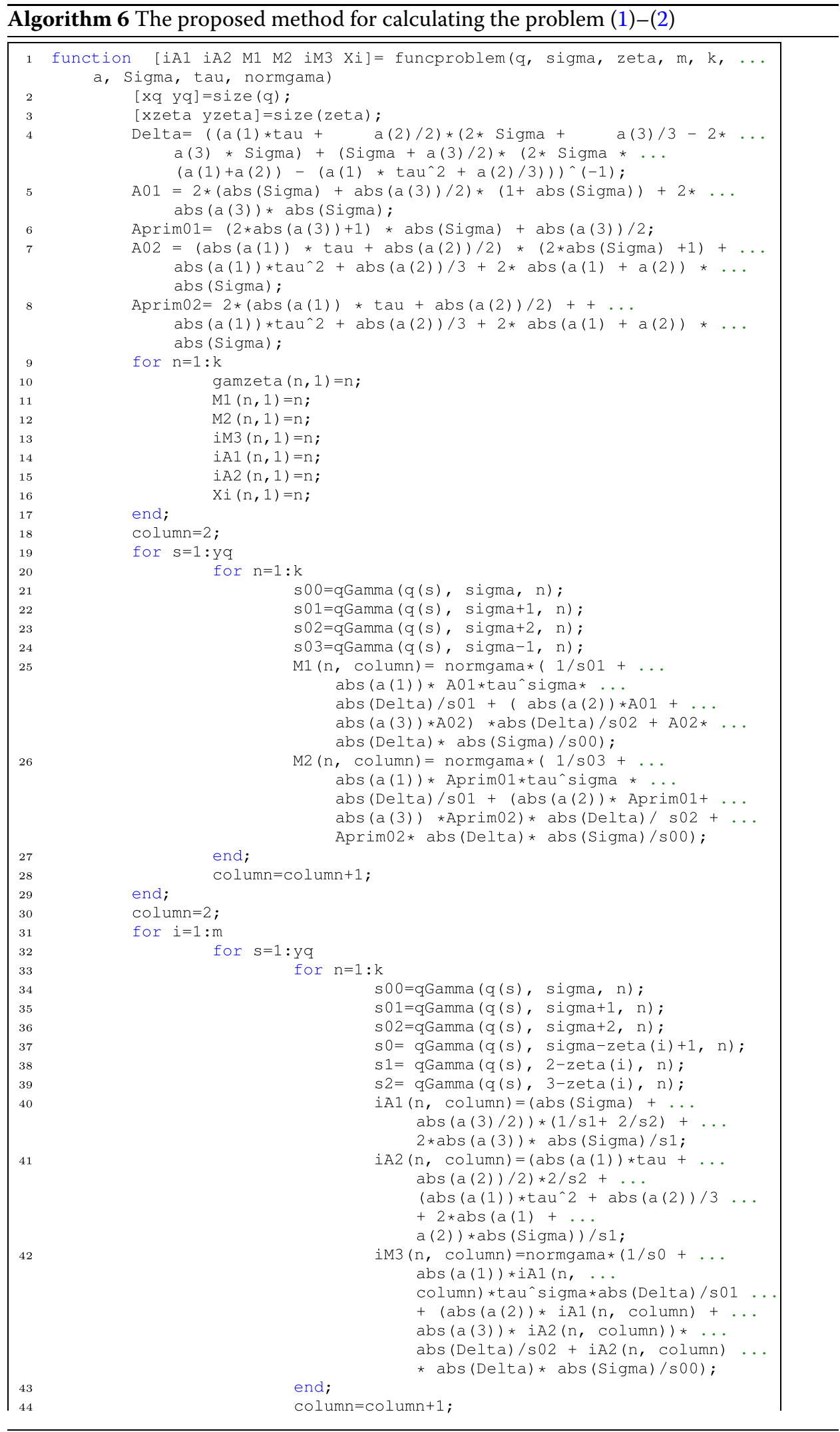



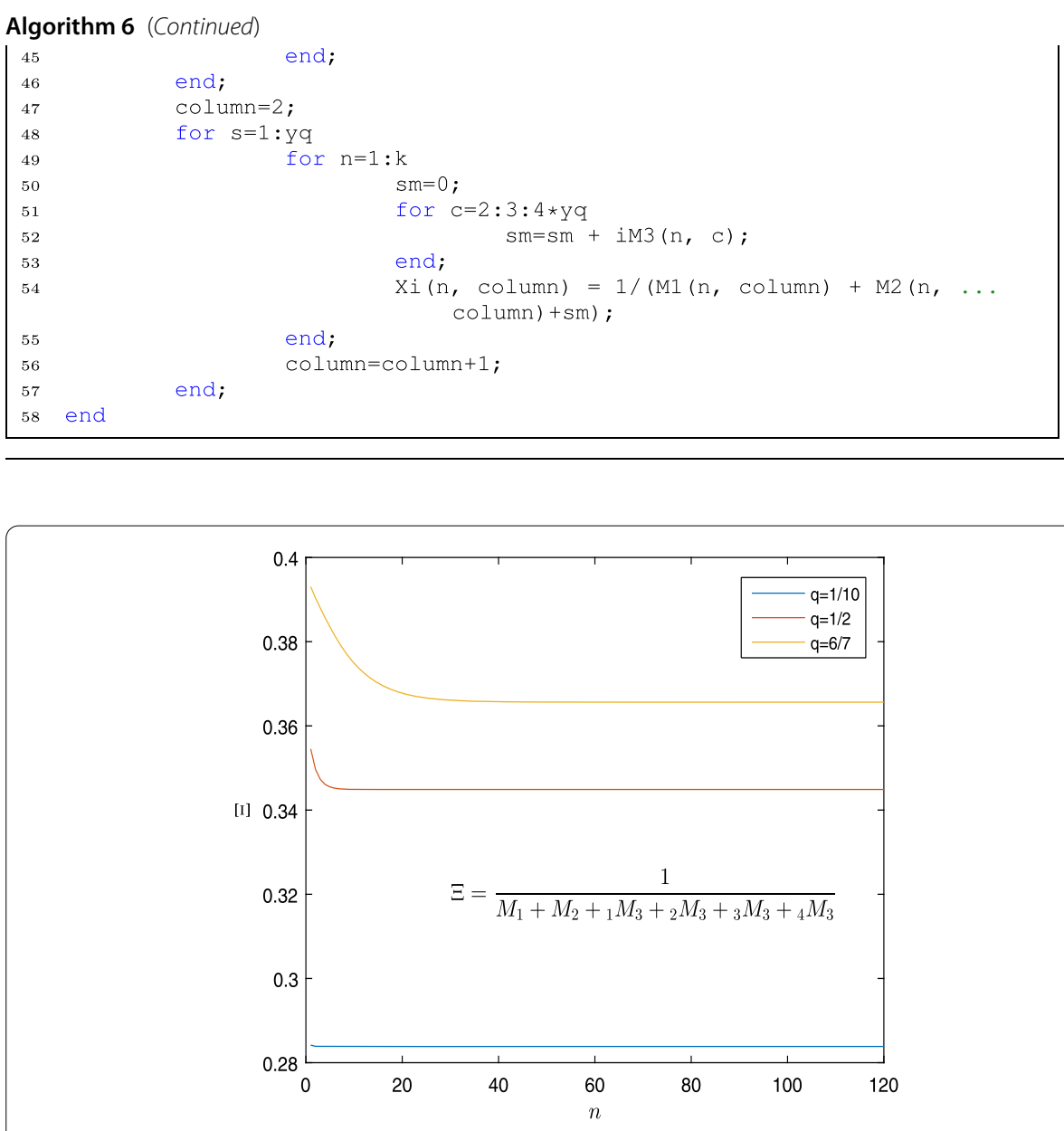

Figure 3 Numerical results of $X i$ where $q=\frac{1}{10}, \frac{1}{2}, \frac{6}{7}$ in Example 1

ability for exact modelings of more phenomena. It is better we concentrate on numerical methods for reviewing of inclusion problems. In this work, by using an endpoint result for set-valued maps, we study a fractional q-differential inclusion problem with sum and integral boundary value conditions on a time scale. We provide an example involving some graphs and algorithms via numerical calculations to illustrate our main result.

\section{Acknowledgements}

The first author was supported by Bu-Ali Sina University. The second author was supported by Azarbaijan Shahid Madani University. The authors express their gratitude to the unknown referees for their helpful suggestions, which improved the final version of this paper.

Funding

Not applicable.

Availability of data and materials

Data sharing not applicable to this article as no datasets were generated or analyzed during the current study.

Ethics approval and consent to participate

Not applicable. 


\section{Consent for publication}

Not applicable.

\section{Authors' contributions}

The authors declare that the study was realized in collaboration with equal responsibility. All authors read and approved the final manuscript.

\section{Author details}

'Department of Mathematics, Bu-Ali Sina University, 65178, Hamedan, Iran. ${ }^{2}$ Institute of Research and Development, Duy Tan University, Da Nang, 550000, Vietnam. ${ }^{3}$ Faculty of Natural Sciences, Duy Tan University, Da Nang, 550000, Vietnam. ${ }^{4}$ Department of Medical Research, China Medical University Hospital, China Medical University, Taichung, Taiwan.

\section{Publisher's Note}

Springer Nature remains neutral with regard to jurisdictional claims in published maps and institutional affiliations.

\section{Received: 7 April 2020 Accepted: 24 August 2020 Published online: 03 September 2020}

\section{References}

1. Jackson, F.H.: q-difference equations. Am. J. Math. 32, 305-314 (1910). https://doi.org/10.2307/2370183

2. Adams, C.R.: The general theory of a class of linear partial q-difference equations. Trans. Am. Math. Soc. 26, 283-312 (1924)

3. Adams, C.R.: Note on the integro-q-difference equations. Trans. Am. Math. Soc. 31(4), 861-867 (1929)

4. Agarwal, R.P.: Certain fractional $q$-integrals and $q$-derivatives. Proc. Camb. Philos. Soc. 66, 365-370 (1969). https://doi.org/10.1017/S0305004100045060

5. Al-Salam, W.A.: q-analogues of Cauchy's formula. Proc. Am. Math. Soc. 17, 182-184 (1952)

6. Ahmad, B., Etemad, S., Ettefagh, M., Rezapour, S.: On the existence of solutions for fractional q-difference inclusions with $q$-antiperiodic boundary conditions. Bull. Math. Soc. Sci. Math. Roum. 59(107), 119-134 (2016). https://doi.org/10.1016/0003-4916(63)90068-X

7. Atici, F., Eloe, P.W.: Fractional $q$-calculus on a time scale. J. Nonlinear Math. Phys. 14(3), 341-352 (2007). https://doi.org/10.2991/jnmp.2007.14.3.4

8. Balkani, N., Rezapour, S., Haghi, R.H.: Approximate solutions for a fractional $q$-integro-difference equation. J. Math. Ext. 13(3), 201-214 (2019)

9. Rezapour, S., Samei, M.E.: On the existence of solutions for a multi-singular pointwise defined fractional q-integro-differential equation. Bound. Value Probl. 2020, 38 (2020). https://doi.org/10.1186/s13661-020-01342-3

10. Samei, M.E., Khalilzadeh Ranjbar, G.: Some theorems of existence of solutions for fractional hybrid $q$-difference inclusion. J. Adv. Math. Stud. 12(1), 63-76 (2019)

11. Samei, M.E., Ranjbar, G.K., Hedayati, V.: Existence of solutions for equations and inclusions of multi-term fractional q-integro-differential with non-separated and initial boundary conditions. J. Inequal. Appl. 2019, 273 (2019). https://doi.org/10.1186/s13660-019-2224-2

12. Ma, C.Y., Shiri, B., Wu, G.C., Baleanu, D.: New fractional signal smoothing equations with short memory and variable order. Optik 218, 164507 (2020). https://doi.org/10.1016/j.ijleo.2020.164507

13. Shiri, B., Wu, G.C., Baleanu, D.: Collocation methods for terminal value problems of tempered fractional differential equations. Appl. Numer. Math. 156, 385-395 (2020). https://doi.org/10.1016/j.apnum.2020.05.007

14. Dadkhah, E., Shiri, B., Ghaffarzadeh, H., Baleanu, D.: Visco-elastic dampers in structural buildings and numerical solution with spline collocation methods. J. Appl. Math. Comput. 63, 29-57 (2020). https://doi.org/10.1007/s12190-019-01307-5

15. Shiri, B., Baleanu, D.: System of fractional differential algebraic equations with applications. Chaos Solitons Fractals 120, 203-212 (2019). https://doi.org/10.1016/j.chaos.2019.01.028

16. Dadkhah, E., Ghaffarzadeh, H., Shiri, B.: Spline collocation methods for seismic analysis of multiple degree of freedom systems with visco-elastic dampers using fractional models. J. Vib. Control 26(17-18), 1445-1462 (2020). https://doi.org/10.1177/1077546319898570

17. Baleanu, D., Shiri, B.: Collocation methods for fractional differential equations involving non-singular kernel. Chaos Solitons Fractals 116, 136-145 (2018). https://doi.org/10.1016/j.chaos.2018.09.020

18. Kumar, D., Singh, J., Baleanu, D.: On the analysis of vibration equation involving a fractional derivative with Mittag-Leffler law. Math. Methods Appl. Sci. 43(1), 443-457 (2019). https://doi.org/10.1002/mma.5903

19. Kumar, D., Singh, J., Tanwar, K., Baleanu, D.: A new fractional exothermic reactions model having constant heat source in porous media with power, exponential and Mittag-Leffler laws. Int. J. Heat Mass Transf. 138, 1222-1227 (2019). https://doi.org/10.1016/j.jijheatmasstransfer.2019.04.094

20. Goswami, A., Singh, J., Kumar, D., Tanwar, K., Sushila: An efficient analytical approach for fractional equal width equations describing hydro-magnetic waves in cold plasma. Phys. A, Stat. Mech. Appl. 524, 563-575 (2019). https://doi.org/10.1016/j.physa.2019.04.058

21. Veeresha, P., Prakasha, D.G., Kumar, D., Baleanu, D., Singh, J.: An efficient computational technique for fractional model of generalized Hirota-Satsuma-coupled Korteweg-de Vries and coupled modified Korteweg-de Vries equations. J. Comput. Nonlinear Dyn. 15(7), 071003 (2020). https://doi.org/10.1115/1.4046898

22. Goswami, A., Sushila, S.J., Kumar, D.: Numerical computation of fractional Kersten-Krasil'shchik coupled kdv-mkdv system occurring in multi-component plasmas. AIMS Math. 5(3), 2346-2368 (2020). https://doi.org/10.3934/math.2020155

23. Baleanu, D., Etemad, S., Rezapour, S.: On a fractional hybrid integro-differential equation with mixed hybrid integral boundary value conditions by using three operators. Alex. Eng. J. (2020). https://doi.org/10.1016/j.aej.2020.04.053

24. Baleanu, D., Rezapour, S., Mohammadi, H.: Some existence results on nonlinear fractional differential equations. Philos. Trans. R. Soc. Lond. Ser. A 2013, 371 (2013). https://doi.org/10.1098/rsta.2012.0144 
25. Agarwal, R.P., Baleanu, D., Hedayati, V., Rezapour, S.: Two fractional derivative inclusion problems via integral boundary conditions. Appl. Math. Comput. 257, 205-212 (2015). https://doi.org/10.1016/j.amc.2014.10.082

26. Baleanu, D., Hedayati, V., Rezapour, S.: On two fractional differential inclusions. SpringerPlus 5, 882 (2016). https://doi.org/10.1186/s40064-016-2564-z

27. Baleanu, D., Aydogan, S.M., Mohammadi, H., Rezapour, S.: On modelling of epidemic childhood diseases with the Caputo-Fabrizio derivative by using the Laplace Adomian decomposition method. Alex. Eng. J. (2020). https://doi.org/10.1016/j.aej.2020.05.007

28. Tuan, N.H., Mohammadi, H., Rezapour, S.: A mathematical model for COVID-19 transmission by using the Caputo fractional derivative. Chaos Solitons Fractals 140, 110107 (2020). https://doi.org/10.1016/j.chaos.2020.110107

29. Baleanu, D., Etemad, S., Rezapour, S.: A hybrid Caputo fractional modeling for thermostat with hybrid boundary value conditions. Bound. Value Probl. 2020, 64 (2020). https://doi.org/10.1186/s13661-020-01361-0

30. Baleanu, D., Jajarmi, A., Mohammadi, H., Rezapour, S.: Analysis of the human liver model with Caputo-Fabrizio fractional derivative. Chaos Solitons Fractals 134, 7 (2020)

31. Ahmad, B., Ntouyas, S.K., Alsedi, A.: On fractional differential inclusions with anti-periodic type integral boundary conditions. Bound. Value Probl. 2013, 82 (2013). https://doi.org/10.1186/1687-2770-2013-82

32. Ghorbanian, R., Hedayati, V., Postolache, M., Rezapour, S.: On a fractional differential inclusion via a new integral boundary condition. J. Inequal. Appl. 2014, 319 (2014). https://doi.org/10.1186/1029-242X-2014-319

33. Agarwal, R.P., Baleanu, D., Hedayati, V., Rezapour, S.: Two fractional derivative inclusion problems via integral boundary condition. Appl. Math. Comput. 257, 205-212 (2015). https://doi.org/10.1016/j.amc.2014.10.082

34. Ntouyas, S.K., Etemad, S.: On the existence of solutions for fractional differential inclusions with sum and integral boundary conditions. Appl. Math. Comput. 266, 235-243 (2015). https://doi.org/10.1016/j.amc.2015.05.036

35. Samei, M.E., Hedayati, V., Rezapour, S.: Existence results for a fraction hybrid differential inclusion with Caputo-Hadamard type fractional derivative. Adv. Differ. Equ. 2019, 163 (2019). https://doi.org/10.1186/s13662-019-2090-8

36. Ntouyas, S.K., Samei, M.E.: Existence and uniqueness of solutions for multi-term fractional $q$-integro-differential equations via quantum calculus. Adv. Differ. Equ. 2019, 475 (2019). https://doi.org/10.1186/s13662-019-2414-8

37. Bohner, M., Peterson, A.: Dynamic Equations on Time Scales. Birkhäuser, Boston (2001)

38. Ernst, T:: A method for q-calculus. J. Nonlinear Math. Phys. 10(4), 487-525 (2003). https://doi.org/10.2991/jnmp.2003.10.4.5

39. Ferreira, R.A.C.: Nontrivials solutions for fractional $q$-difference boundary value problems. Electron. J. Qual. Theory Differ. Equ. 2010, 70 (2010)

40. Amini-Harandi, A.: Endpoints of set-valued contractions in metric spaces. Nonlinear Anal. 72(1), 132-134 (2010). https://doi.org/10.1016/j.na.2009.06.074

41. Kisielewicz, M.: Differential Inclusions and Optimal Control. Kluwer, Dordrecht (1991)

42. Covitz, H., Nadler, S.: Multivalued contraction mappings in generalized metric spaces. Isr. J. Math. 8, 5-11 (1970)

43. Aubin, J., Ceuina, A.: Differential Inclusions: Set-Valued Maps and Viability Theory. Springer, Berlin (1984). https://doi.org/10.1007/978-3-642-69512-4

44. Deimling, K.: Multi-Valued Differential Equations. de Gruyter, Berlin (1992)

45. Berinde, V., Pacurar, M.: The role of the Pompeiu-Hausdorff metric in fixed point theory. Creative Math. Inform. 22(2), 143-150 (2013)

46. El-Sayed, A.M.A., Ibrahim, A.G.: Multivalued fractional differential equations. Appl. Math. Comput. 68, 15-25 (1995). https://doi.org/10.1016/0096-3003(94)00080-N

47. Lasota, A., Opial, Z: An application of the Kakutani-Ky Fan theorem in the theory of ordinary differential equations. Bull. Acad. Pol. Sci., Sér. Sci. Math. Astron. Phys. 13, 781-786 (1965)

48. Podlubny, l.: Fractional Differential Equations. Academic Press, San Diego (1999)

\section{Submit your manuscript to a SpringerOpen ${ }^{\circ}$ journal and benefit from:}

- Convenient online submission

- Rigorous peer review

- Open access: articles freely available online

- High visibility within the field

- Retaining the copyright to your article

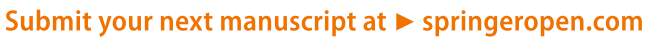

